\title{
EXISTENCE AND UNIQUENESS OF LOW-ENERGY WEAK SOLUTIONS TO THE COMPRESSIBLE 3D MAGNETOHYDRODYNAMICS EQUATIONS
}

\author{
ANTHONY SUEN \\ To my family and my daughter Elisa
}

\begin{abstract}
We prove the existence and uniqueness of weak solutions of the three dimensional compressible magnetohydrodynamics (MHD) equations. We first obtain the existence of weak solutions with small $L^{2}$-norm which may display codimension-one discontinuities in density, pressure, magnetic field and velocity gradient. The weak solutions we consider here exhibit just enough regularity and structure which allow us to develop uniqueness and continuous dependence theory for the compressible MHD equations. Our results generalise and extend those for the intermediate weak solutions of compressible NavierStokes equations.
\end{abstract}

\section{INTRODUCTION}

Magnetohydrodynamics (MHD) studies the dynamics of electrically conducting fluids under the influence of magnetic fields. There are many examples of conducting fluids, including plasmas, liquid metals, electrolytes, etc. The main idea of magnetohydrodynamics is that conducting fluids can support magnetic fields. More precisely, magnetic fields can induce currents in a moving conducting fluid, which in turn create forces on the fluid and also change the magnetic fields themselves. The subject of magnetohydrodynamics unites classical fluid dynamics with electrodynamics, and references can be found in [1, 4, [5], 6].

In this present work, we focus on the following compressible barotropic model:

$$
\begin{aligned}
\rho_{t}+\operatorname{div}(\rho u) & =0, \\
\left(\rho u^{j}\right)_{t}+\operatorname{div}\left(\rho u^{j} u\right)+P(\rho)_{x_{j}}+\left(\frac{1}{2}|B|^{2}\right)_{x_{j}}-\operatorname{div}\left(B^{j} B\right) & =\mu \Delta u^{j}+\lambda \operatorname{div} u_{x_{j}}, \\
B_{t}^{j}+\operatorname{div}\left(B^{j} u-u^{j} B\right) & =\nu \Delta B^{j}, \\
\operatorname{div}(B) & =0
\end{aligned}
$$

with given initial data

$$
(\rho, u, B)(x, 0)=\left(\rho_{0}, u_{0}, B_{0}\right)(x) .
$$

For our barotropic model, the temperature is taken to be constant and the state of fluid motion is specified by three physical quantities: density $\rho$, velocity $u=$ $\left(u^{1}, u^{2}, u^{3}\right) \in \mathbb{R}^{3}$ and magnetic field $B=\left(B^{1}, B^{2}, B^{3}\right) \in \mathbb{R}^{3}$. These quantities are

Date: June 30, 2019.

2000 Mathematics Subject Classification. 35Q35, 35Q80.

Key words and phrases. compressible magnetohydrodynamics, global weak solutions, uniqueness, continuous dependence. 
all functions of the spatial coordinate $x \in \mathbb{R}^{3}$ and time $t \geq 0 . P=P(\rho)$ is the pressure which is an increasing function of $\rho . \mu, \lambda$ are positive viscosity coefficients and $\nu$ is the magnetic diffusivity. For a compressible barotropic flow, the equations of dynamics are given by the Navier-Stokes equations (1.1)-(1.2) which express the conservation of mass and conservation of momentum respectively. On the other hand, Maxwell's equations (1.3)-(1.4) govern the electromagnetic phenomena of the conducting fluid and the dynamics of magnetic fields. Therefore, by combining the compressible Navier-Stokes equations with Maxwell's equations, we obtain equations (1.1)-(1.4) which model the macroscopic behavior of electrically conducting fluids. We refer to Cabannes [4, Biskamp [3] and Freistühler [9] for more detailed discussions and derivation of the system (1.1)-(1.4).

The global well-posedness of the system (1.1)-(1.4) is an active topic in mathematics, and the cases $\nu>0$ and $\nu=0$ are both of interest. When the magnetic diffusivity $\nu$ is taken to be positive, different types of solutions to (1.1)-(1.4) are proved to exist for all time:

(a) The first type of solutions to (1.1)-(1.4) are the small-smooth solutions. More precisely, Kawashima [19] proved the global-in-time existence of $H^{3}$ solutions for (1.1)-(1.4) when the initial data was taken to be small in $H^{3}$ modulo a constant state. His analysis consists an iterative procedure based on asymptotic decay rates for the corresponding linearised equations. The major weakness of small-smooth solutions is that they do not exhibit nonlinear effects and tell us relatively little about the fluid flow.

(b) The second type of solutions to (1.1)-(1.4) are the large-energy weak solutions. In this category, solutions are proved to exist for initial data with arbitrarily large energy and nonnegative density, which can be achieved by showing that sequences of approximate solutions with uniform energy estimates and entropy estimates have strongly converging subsequences. These results were obtained by $\mathrm{Hu}$ and Wang [17-[18] and Sart [20] which generalised the previous results proved by Lions [16] and Feireisl [7-8] for compressible Navier-Stokes system. Large-energy weak solutions by their very nature possess very little regularity, which may even include some non-physical solutions (see [13] and 14 for related discussions).

(c) Apart from those two types of solutions as mentioned in (a) and (b), Suen and Hoff [22] proved the global-in-time existence of intermediate weak solutions which was an extension of the intermediate regularity class of solutions for compressible Navier-Stokes system introduced by Hoff [10]-[12]. Such intermediate regularity class of solutions has rich physical and mathematical meanings compared to other solution classes. In this category, initial data is assumed to be small in some weak norms $\left(L^{2}\right)$ with nonnegative and essentially bounded initial densities. From the results obtained by Hoff and Santos [15] for the Navier-Stokes system, it can be seen that solutions may exhibit discontinuities in density and velocity gradient across hypersurfaces in $\mathbb{R}^{2}$ or $\mathbb{R}^{3}$, which is not observable from small-smooth solutions mentioned in (a). On the other hand, the solutions would still have enough regularity for the development of a uniqueness and continuous dependence theory [12] which seems unreachable within or from the very weak framework used by Lions and Feireisl mentioned in (b).

In light of (c) as described above, the main goal of the present work is therefore to address the global-in-time existence and uniqueness of intermediate weak solutions of the system (1.1)-(1.4). The novelties of this current work are as follows: 
1. We strengthen the results obtained in Suen-Hoff [22, in the way that we show the details of the $s$-dependence of various smoothing rates near $t=0$ resulting from the hypothesis that $u_{0}, B_{0} \in H^{s}$ for $s \in\left(\frac{1}{2}, 1\right]$. Such regularity requirement on $u_{0}, B_{0}$ is crucial in obtaining uniqueness of the weak solutions of (1.1)-(1.4). It also matches with the results given in Hoff 11 for Navier-Stokes equations.

2. We obtain new estimates on various auxiliary functionals which are important in controlling the strong coupling effects between density, velocity and magnetic fields. Those estimates will be used in proving both existence and uniqueness of weak solutions of (1.1)-(1.4).

3. We successfully extend the uniqueness and continuous dependence theory given in [12] for compressible Navier-Stokes system to compressible MHD system (1.1)(1.4).

We give a brief exposition on the analysis applied in this work. First of all, we introduce an important canonical variable associated with the system (1.1)-(1.4), which is known as the effective viscous flux. To see how it works, by the Helmholtz decomposition of the mechanical forces, we can rewrite the momentum equation (1.2) as follows (summation over $k$ is understood):

$$
\rho \dot{u}^{j}+\left(\frac{1}{2}|B|^{2}\right)_{x_{j}}-\operatorname{div}\left(B^{j} B\right)=F_{x_{j}}+\mu \omega_{x_{k}}^{j, k},
$$

where $\dot{u}^{j}=u_{t}^{j}+u \cdot u^{j}$ is the material derivative on $u^{j}$ and the effective viscous flux $F$ is defined by

$$
F=(\mu+\lambda) \operatorname{div}(u)-P(\rho)+P(\tilde{\rho}) .
$$

Differentiating (1.6), we obtain the following Poisson equation

$$
\Delta F=\operatorname{div}(g),
$$

where $g^{j}=\rho \dot{u}^{j}+\left(\frac{1}{2}|B|^{2}\right)_{x_{j}}-\operatorname{div}\left(B^{j} B\right)$. This Poisson equation (1.8) is thus the analog for compressible MHD of the well-known elliptic equation for pressure in incompressible flow. The effective viscous flux $F$ plays a crucial roll in the overall analysis:

1. The equation (1.6) expresses the acceleration density $\rho \dot{u}$ as the sum of the gradient of the scalar $F$ and the divergence-free vector field $\omega_{x_{k}}^{, k}$, modulo lowerorder terms involving $B$. The skew-symmetry of $\omega$ insures that these two vector fields are orthogonal in $L^{2}\left(\mathbb{R}^{3}\right)$, so that $L^{2}$-bounds for the terms on the left side of (1.6) immediately give $L^{2}$ bounds for the gradients of both $F$ and $\omega$. These in turn will be used for controlling $\nabla u$ in $L^{4}$ when $u(\cdot, t) \notin H^{2}$.

2. With the help of the effective viscous flux $F$ on the mass equation (1.1), we can further rewrite the equation as follows:

$$
(\mu+\lambda) \frac{d}{d t}[\log \rho(x(t), t)-\log (\tilde{\rho})]+P(\rho(x(t), t))-\tilde{P}=-F(x(t), t),
$$

where $x(t)$ is an integral curve of $u$ and $\tilde{\rho}$ is some constant density. Upon integrating the above equation with respect to $t$ on some interval $\left[t_{1}, t_{2}\right]$, if $P$ is increasing, then the integral of $P-\tilde{P}$ on the left side gives a dissipative term. Hence it suffices to control the term $\int_{t_{0}}^{t_{1}} F(x(\tau), \tau) d \tau$. If $\Gamma$ is the fundamental solution for the Laplace operator on $\mathbb{R}^{3}$, then from (1.8) we have

$$
F=\Gamma_{x_{j}} *\left(\rho \dot{u}^{j}+\left(\frac{1}{2}|B|^{2}\right)_{x_{j}}-\operatorname{div}\left(B^{j} B\right)\right) .
$$


There is a cancellation between the material derivative on $u$ and the time integral in $\int_{t_{0}}^{t_{1}} F(s) d s$, as a result we can obtain integrals in lower regularity and hence greater integrability in time. Such observation is essential in proving the pointwise bounds on the density, which allows us to obtain sufficient a priori bounds on the solutions.

3. One of the key step in proving uniqueness of weak solutions is to obtain a bound on $\int_{0}^{t}\|\nabla u(\cdot, \tau)\|_{L^{\infty}} d \tau$. Our attempt is to decompose $u$ as $u=u_{F}+u_{P}$, where $u_{F}$, $u_{P}$ satisfy

$$
\left\{\begin{array}{l}
(\mu+\lambda) \Delta u_{F}^{j}=F_{x_{j}}+(\mu+\lambda) \omega_{x_{k}}^{j, k} \\
(\mu+\lambda) \Delta u_{P}^{j}=(P-P(\tilde{\rho}))_{x_{j}}
\end{array}\right.
$$

Using the a priori bounds on the effective viscous flux $F$, we can bound the integral $\int_{0}^{t}\left\|\nabla u_{F}(\cdot, \tau)\right\|_{L^{\infty}} d \tau$ in terms of $F$. On the other hand, to bound the integral $\int_{0}^{t}\left\|\nabla u_{P}(\cdot, \tau)\right\|_{\infty} d \tau$, we point out that $(\rho-\tilde{\rho}) \in L^{2} \cap L^{\infty}$ is not sufficient for bounding $\left\|\nabla u_{P}(\cdot, \tau)\right\|_{\infty}$. However, if $P(\rho(\cdot, t)) \in L^{\infty}$, then $u_{P}^{j}(\cdot, t)=(\mu+\lambda)^{-1} \Gamma_{x_{j}} *$ $(P(\rho(\cdot, t))-\tilde{P})$ is log-Lipschitz. This is sufficient to guarantee that the integral curve $x(\cdot, t)$ of $u=u_{F}+u_{P}$ (assuming that $u_{F}$ has enough regularity as claimed) is Hölder continuous. If we assume that the initial density is piecewise Hölder continuous, then using the mass equation (1.1), it implies that the density is also piecewise Hölder continuous for positive time. Hence with such improved regularity on the density, it allows us to obtain the desired bound on $\int_{0}^{t}\left\|\nabla u_{P}(\cdot, \tau)\right\|_{\infty} d \tau$.

We now give a detailed formulation of our results. To begin with, we require that the viscosity constants $\mu, \lambda, \nu$ and pressure $P(\rho)$ satisfy

$$
\mu, \lambda, \nu>0, \quad \frac{\mu}{\lambda}>4
$$

and

$$
P^{\prime}(\rho)>0, \rho>0
$$

The weak solutions to (1.1)-(1.4) are defined as follows.

Definition 1.1. We let $\tilde{\rho}$ be a fixed, positive, constant reference density and we take $\tilde{P}=P(\tilde{\rho})$. The weak solutions we study in this paper are defined as follows. $A$ weak solution of the system (1.1)-(1.4) is a triple $(\rho, u, B)$ which satisfies

- $(\rho-\tilde{\rho}, \rho u, B) \in C\left([0, \infty) ; H^{-1}\left(\mathbb{R}^{3}\right)\right)$ with $\left.(\rho, u, B)\right|_{t=0}=\left(\rho_{0}, u_{0}, B_{0}\right)$;

- $\nabla u, \nabla B \in L^{2}\left(\mathbb{R}^{3} \times(0, \infty)\right)$;

- $\operatorname{div}(B)(\cdot, t)=0$ in $\mathcal{D}^{\prime}\left(\mathbb{R}^{3}\right)$ for $t>0$;

and the following identities hold for times $t_{2} \geq t_{1} \geq 0$ and $C^{1}$ test functions $\varphi$ having uniformly bounded support in $x$ for $t \in\left[t_{1}, t_{2}\right]$ :

$$
\left.\int_{\mathbb{R}^{3}} \rho(x, \cdot) \varphi(x, \cdot) d x\right|_{t_{1}} ^{t_{2}}=\int_{t_{1}}^{t_{2}} \int_{\mathbb{R}^{3}}\left(\rho \varphi_{t}+\rho u \cdot \nabla \varphi\right) d x d \tau
$$




$$
\begin{aligned}
\left.\int_{\mathbb{R}^{3}}\left(\rho u^{j}\right)(x, \cdot) \varphi(x, \cdot) d x\right|_{t_{1}} ^{t_{2}}=\int_{t_{1}}^{t_{2}} & \int_{\mathbb{R}^{3}}\left[\rho u^{j} \varphi_{t}+\rho u^{j} u \cdot \nabla \varphi+P(\rho) \varphi_{x_{j}}\right] d x d \tau \\
& +\int_{t_{1}}^{t_{2}} \int_{\mathbb{R}^{3}}\left[\frac{1}{2}|B|^{2} \varphi_{x_{j}}-B^{j} B \cdot \nabla \varphi\right] d x d \tau \\
& -\int_{t_{1}}^{t_{2}} \int_{\mathbb{R}^{3}}\left[(\mu+\lambda) \nabla u^{j} \cdot \nabla \varphi+\lambda(\operatorname{div}(u)) \varphi_{x_{j}}\right] d x d \tau
\end{aligned}
$$

and

$$
\left.\int_{\mathbb{R}^{3}} B^{j}(x, \cdot) \varphi(x, \cdot) d x\right|_{t_{1}} ^{t_{2}}=\int_{t_{1}}^{t_{2}} \int_{\mathbb{R}^{3}}\left[\left(B^{j} u-u^{j} B\right) \cdot \nabla \varphi-\nu \nabla B^{j} \cdot \nabla \varphi\right] d x d \tau .
$$

We adopt the usual notation for Hölder seminorms, namely for $v: \mathbb{R}^{3} \rightarrow \mathbb{R}^{3}$ and $\alpha \in(0,1]$,

$$
\langle v\rangle^{\alpha}=\sup _{\substack{x_{1}, x_{2} \in \mathbb{R}^{3} \\ x_{1} \neq x_{2}}} \frac{\left|v\left(x_{2}\right)-v\left(x_{1}\right)\right|}{\left|x_{2}-x_{1}\right|^{\alpha}} ;
$$

and for $v: Q \subseteq \mathbb{R}^{3} \times[0, \infty) \rightarrow \mathbb{R}^{3}$ and $\alpha_{1}, \alpha_{2} \in(0,1]$

$$
\langle v\rangle_{Q}^{\alpha_{1}, \alpha_{2}}=\sup _{\substack{\left(x_{1}, t_{1}\right),\left(x_{2}, t_{2}\right) \in Q \\\left(x_{1}, t_{1}\right) \neq\left(x_{2}, t_{2}\right)}} \frac{\left|v\left(x_{2}, t_{2}\right)-v\left(x_{1}, t_{1}\right)\right|}{\left|x_{2}-x_{1}\right|^{\alpha_{1}}+\left|t_{2}-t_{1}\right|^{\alpha_{2}}} .
$$

We give the definition of piecewise Hölder continuous as follows. We also refer to Hoff 11 for more details.

Definition 1.2. We say that a function $\phi(\cdot, t)$ is piecewise $C^{\beta(t)}$ if it has simple discontinuities across a $C^{\beta(t)+1}$ curve $\mathcal{C}(t): \mathcal{C}(t)=\{y(s, t): s \in I \subset \mathbb{R}\}$, where $\beta(t)>0$ is a function in $t, I$ is an open interval and the curve $\mathcal{C}(t)$ is the u-transport of $\mathcal{C}(0)$ given by:

$$
y(s, t)=y(s, 0)+\int_{0}^{t} u(y(s, \tau), \tau) d \tau .
$$

Here $\mathcal{C}(0)$ is a $C^{\beta_{0}+1}$ curve with $\beta(0)=\beta_{0}>0$, which means that

$$
\mathcal{C}(0)=\left\{y_{0}(s): s \in \mathbb{R}\right\},
$$

where $y(s, 0)=y_{0}(s)$ is parameterised in arc length $s$ and $y_{0}$ is $C^{\beta_{0}+1}$. The complement of $\mathcal{C}(0)$ consists of two disjoint, connected, open sets $\Omega_{+}(0)$ and $\Omega_{-}(0)$ with $\mathcal{C}(0)=\partial \Omega_{ \pm}(0)$.

We denote the norm $\|\phi(\cdot, t)\|_{C_{p w}^{\beta(t)}}$ by

$$
\|\phi(\cdot, t)\|_{C_{p w}^{\beta(t)}}=\|\phi(\cdot, t)\|_{L^{\infty}}+\sup _{x_{1} \neq x_{2}} \frac{\left|\phi\left(x_{2}, t\right)-\phi\left(x_{1}, t\right)\right|}{\left|x_{2}-x_{1}\right|^{\beta(t)}},
$$

where the supremum is taken over points $x_{1}, x_{2}$ on the same side of $\mathcal{C}(t)$.

We also make use of the following standard facts (see Ziemer [23. Theorem 2.1.4, Remark 2.4.3, and Theorem 2.4.4], for example):

- First, given $r \in[2,6]$ there is a constant $C(r)$ such that for $w \in H^{1}\left(\mathbb{R}^{3}\right)$,

$$
\|w\|_{L^{r}\left(\mathbb{R}^{3}\right)} \leq C(r)\left(\|w\|_{L^{2}\left(\mathbb{R}^{3}\right)}^{(6-r) / 2 r}\|\nabla w\|_{L^{2}\left(\mathbb{R}^{3}\right)}^{(3 r-6) / 2 r}\right)
$$

and

where $\alpha=1-3 / r$;

$$
\langle w\rangle^{\alpha} \leq C(r)\|\nabla w\|_{L^{r}\left(\mathbb{R}^{3}\right)},
$$


- for any $r \in(3, \infty)$ there is a constant $C(r)$ such that for $w \in W^{1, r}\left(\mathbb{R}^{3}\right)$,

$$
\|w\|_{L^{\infty}\left(\mathbb{R}^{3}\right)} \leq C(r)\|w\|_{W^{1, r}\left(\mathbb{R}^{3}\right)} .
$$

We now state our main results. Theorem 1.3 gives the existence of weak solutions to (1.1)-(1.4) with the $s$-dependence of smooth rates near $t=0$ (see (1.26) below). When the initial density $\rho_{0}$ is piecewise $C^{\beta_{0}}$ for some $\beta_{0}>0$ as defined in Definition [1.2, we prove that $\rho(\cdot, t)$ is piecewise $C^{\beta(t)}$ for $\beta(t) \in\left(0, \beta_{0}\right]$, which provides sufficient regularity in obtaining the bound on the time integral of $\|\nabla u(\cdot, t)\|_{L^{\infty}}$.

Theorem 1.3. Fix constants $L, \rho_{1}, \rho_{2}, \tilde{\rho}>0, q>6$ and $s \in\left(\frac{1}{2}, 1\right]$ and assume that $\mu, \lambda, \nu, P$ satisfy (1.9)-(1.10). There exists positive constants $d, \theta, C$ such that if the initial data $\left(\rho_{0}, u_{0}, B_{0}\right)$ is given satisfying

$$
\begin{gathered}
\rho_{1} \leq \rho_{0}(x) \leq \rho_{2}, x \in \mathbb{R}^{3}, \\
\left\|u_{0}\right\|_{L^{q}}+\left\|B_{0}\right\|_{L^{q}} \leq L \\
C_{0}=\left\|u_{0}\right\|_{H^{s}}^{2}+\left\|B_{0}\right\|_{H^{s}}^{2}+\int_{\mathbb{R}^{3}}\left(|\rho-\tilde{\rho}|^{2}+\left|u_{0}\right|^{2}+\left|B_{0}\right|^{2}\right) d x \leq d,
\end{gathered}
$$

then the system (1.1) -(1.4) has a global weak solution $(\rho, u, B)$ in the sense of (1.11) (1.13) on all of $\mathbb{R}^{3} \times[0, \infty)$. The solution satisfies the following:

$$
\begin{gathered}
\rho-\tilde{\rho} \in C\left([0, \infty) ; H^{-1}\left(\mathbb{R}^{3}\right)\right) ; \\
u, B \in C\left([0, \infty) ; L^{2}\left(\mathbb{R}^{3}\right)\right) ; \\
\nabla u, \nabla B \in L^{2}\left(\mathbb{R}^{3} \times(0, \infty)\right) ; \\
u(\cdot, t), B(\cdot, t) \in H^{1}\left(\mathbb{R}^{3}\right), t>0 ; \\
\langle u\rangle_{\mathbb{R}^{3} \times[\tau, \infty)}^{\frac{1}{2}, \frac{1}{4}},\langle B\rangle_{\mathbb{R}^{3} \times[\tau, \infty)}^{\frac{1}{2}, \frac{1}{4}} \leq C(t) C_{0}^{\theta}, t>0,
\end{gathered}
$$

where $C(\tau)$ may depend additionally on a positive lower bound for $\tau$, and the following bounds hold:

$$
\begin{gathered}
\frac{1}{2} \rho_{1} \leq \rho(x, t) \leq 2 \rho_{2} \\
\sup _{t>0} \int_{\mathbb{R}^{3}}\left(|\rho-\tilde{\rho}|^{2}+|u|^{2}+|B|^{2}+\sigma^{1-s}\left(|\nabla u|^{2}+|\nabla B|^{2}\right)+\sigma^{2-s}\left(|\dot{u}|^{2}+\left|B_{t}\right|^{2}\right)\right) d x \\
+\int_{0}^{\infty} \int_{\mathbb{R}^{3}}\left(|\nabla u|^{2}+|\nabla B|^{2}+\sigma^{1-s}\left(|\dot{u}|^{2}+\left|B_{t}\right|^{2}\right)+\sigma^{2-s}\left(|\nabla \dot{u}|^{2}+\left|\nabla B_{t}\right|^{2}\right)\right) d x d \tau \\
\leq C C_{0}^{\theta},
\end{gathered}
$$

where $\dot{u}=u_{t}+\nabla u \cdot u$ is the material derivative of $u$ and $\sigma=\min \{1, t\}$.

Furthermore, for $\beta_{0}>0$, given a $C^{\beta_{0}+1}$ curve $y_{0}$ as described in Definition 1.2, if there exists $N>0$ such that

$$
\left\|\rho_{0}(\cdot)-\tilde{\rho}\right\|_{C_{p w}^{\beta_{0}}} \leq N
$$

then for each $T>0$ and $t \in[0, T]$, there are $\beta(t) \in\left(0, \beta_{0}\right]$ and $C\left(N, T, C_{0}\right)>0$ such that we have

$$
\sup _{0 \leq \tau \leq T}\|\rho(\cdot, t)-\tilde{\rho}\|_{C_{p w}^{\beta(t)}}+\int_{0}^{T}\|\nabla u(\cdot, \tau)\|_{L^{\infty}} d \tau \leq C\left(N, T, C_{0}\right) .
$$


Remark 1.4. We point out that the piecewise $C^{\beta_{0}}$-norm of $\rho_{0}$ is required to be bounded but not necessary small. This is different from the case of Hoff [11, in which the author imposed a smallness assumption on $\left\|\rho_{0}\right\|_{C_{p w}^{\beta_{0}}}$ and proved that $\|\rho(\cdot, t)\|_{C_{p w}^{\beta_{0}}}$ remains small for all $t>0$. The key observation in our present work is that, without the smallness assumption on $\left\|\rho_{0}\right\|_{C_{p w}^{\beta_{0}}}$, we are able to show that there exists $\beta(t) \in\left(0, \beta_{0}\right]$ such that $\|\rho(\cdot, t)\|_{C_{p w}^{\beta(t)}}$ remains finite for finite time.

Once we obtain Theorem 1.3. we address the uniqueness of weak solutions given in Theorem 1.3 which can be summarised as follows. Theorem 1.5] illustrates the continuous dependence on the initial data of weak solutions, which generalises the results in Hoff [12] for compressible Navier-Stokes equations.

Theorem 1.5. Fix constants $N, L, \rho_{1}, \rho_{2}, \tilde{\rho}>0, q>6$ and $s \in\left(\frac{1}{2}, 1\right]$ and assume that $\mu, \lambda$ satisfy (1.9) and $P$ satisfies

$$
P(\rho)=K \rho
$$

for some constant $K$. Assume that $\left(\rho_{0}, u_{0}, B_{0}\right)$ and $\left(\bar{\rho}_{0}, \bar{u}_{0}, \bar{B}_{0}\right)$ are functions satisfying (1.17)-(1.19) and (1.27) as in Theorem 1.3. Then for each $T>0$, there exists $C(T)>0$ such that if $(\rho, u, B)$ and $(\bar{\rho}, \bar{u}, \bar{B})$ are weak solutions to (1.1)-(1.4) as described in Theorem 1.3 with initial data $\left(\rho_{0}, u_{0}, B_{0}\right)$ and $\left(\bar{\rho}_{0}, \bar{u}_{0}, \bar{B}_{0}\right)$ respectively, then we have

$$
\begin{aligned}
& \left(\int_{0}^{T} \int_{\mathbb{R}^{3}}\left(|u-\bar{u}|^{2}+|B-\bar{B}|^{2}\right) d x d \tau\right)^{\frac{1}{2}}+\sup _{0 \leq \tau \leq T}\|(\rho-\bar{\rho})(\cdot, t)\|_{H^{-1}} \\
& \leq C(T)\left[\left\|\rho_{0}-\bar{\rho}_{0}\right\|_{L^{2}}+\left\|\rho_{0} u_{0}-\bar{\rho}_{0} \bar{u}_{0}\right\|_{L^{2}}+\left\|B_{0}-\bar{B}_{0}\right\|_{L^{2}}\right] .
\end{aligned}
$$

Remark 1.6. Similar to the case as in Hoff [12, the condition (1.29) on the pressure can be replaced by a more general one, namely

$$
\sup _{0 \leq \tau \leq T}\left\|\nabla\left(\frac{P(\rho(\cdot, t))-P(\bar{\rho}(\cdot, t))}{\rho(\cdot, t)-\bar{\rho}(\cdot, t)}\right)\right\|_{L^{3}}<\infty .
$$

The rest of the paper is organised as follows. In Section 2, we obtain a priori estimates for smooth solutions to (1.1)-(1.4). In Section 3 we apply the estimates obtained in Section 2 to prove Theorem 1.3 and give the details in obtaining bound on the time integral of $\|\nabla u(\cdot, t)\|_{L^{\infty}}$. Finally in Section 4 , we address the uniqueness of weak solutions given in Theorem 1.3 by making use of the Lagrangian coordinates (the integral curve of $u$ ) and bounds on some auxiliary functionals.

\section{A PRIORI ESTimates}

In this section, we obtain some a priori estimates for smooth local-in-time solutions $(\rho-\tilde{\rho}, u, B)$ of (1.1)-(1.4). We first recall the following local-in-time existence theorem which was proved by Kawashima [19]:

Theorem 2.1. For a given initial data $\left(\rho_{0}-\tilde{\rho}, u_{0}, B_{0}\right) \in H^{3}\left(\mathbb{R}^{3}\right)$, there exists $T>0$ and a solution $(\rho, u, B)$ to (1.1)-(1.4) defined on $\mathbb{R}^{3} \times[0, T]$ such that

$$
\rho-\tilde{\rho} \in C\left([0, T] ; H^{3}\left(\mathbb{R}^{3}\right)\right) \cap C^{1}\left([0, T] ; H^{2}\left(\mathbb{R}^{3}\right)\right)
$$

and

$$
u, B \in C\left([0, T] ; H^{3}\left(\mathbb{R}^{3}\right)\right) \cap C^{1}\left([0, T] ; H^{1}\left(\mathbb{R}^{3}\right)\right) \cap L^{2}\left([0, T] ; H^{4}\left(\mathbb{R}^{3}\right)\right) .
$$


The estimates for $(\rho-\tilde{\rho}, u, B)$ given in this section will be crucial in proving Theorem 1.3. The main goal is to prove the following theorem:

Theorem 2.2. Fix constants $L, \rho_{1}, \rho_{2}, \tilde{\rho}>0, q>6$ and $s \in\left(\frac{1}{2}, 1\right]$, and assume that $\mu, \lambda, P$ satisfy (1.9)-(1.10). Let the initial data $\left(\rho_{0}-\tilde{\rho}, u_{0}, B_{0}\right) \in H^{3}\left(\mathbb{R}^{3}\right)$ be given satisfying (1.17)-(1.19). There exists positive constants $d, \theta, C$ such that if $(\rho, u, B)$ is a solution of (1.1)-(1.4) on $\mathbb{R}^{3} \times[0, T]$ satisfying (2.1)-(2.2), then we have

$$
\mathcal{A}(T) \leq C C_{0}^{\theta}
$$

and

$$
\frac{1}{2} \rho_{1} \leq \rho(x, t) \leq 2 \rho_{2},(x, t) \in \mathbb{R}^{3} \times[0, T],
$$

where $\mathcal{A}(T)$ is given by

$$
\begin{aligned}
\mathcal{A}(T) & =\sup _{0 \leq \tau \leq T}\left[\int_{\mathbb{R}^{3}}\left(|\rho-\tilde{\rho}|^{2}+|u|^{2}+|B|^{2}\right)\right](x, \tau) d x \\
& +\sup _{0 \leq \tau \leq T}\left[\int_{\mathbb{R}^{3}} \sigma^{1-s}\left(|\nabla u|^{2}+|\nabla B|^{2}\right)+\sigma^{2-s}\left(|\dot{u}|^{2}+\left|B_{t}\right|^{2}\right)\right](x, \tau) d x \\
& +\int_{0}^{T} \int_{\mathbb{R}^{3}}\left(|\nabla u|^{2}+|\nabla B|^{2}+\sigma^{1-s}\left(|\dot{u}|^{2}+\left|B_{t}\right|^{2}\right)+\sigma^{2-s}\left(|\nabla \dot{u}|^{2}+\left|\nabla B_{t}\right|^{2}\right)\right) d x d \tau,
\end{aligned}
$$

and $\sigma(t)=\min \{1, t\}$.

The proof of Theorem 2.2 will be carried out in a sequence of lemmas. We first establish the bound (2.3) under the assumption that (2.4) holds for the density $\rho$, which will be given in subsection 2.1 and subsection 2.2. Then in subsection 2.3. we close the estimates of Theorem 2.2 by deriving pointwise bounds (2.4) for $\rho$ under the smallness assumption on $C_{0}$. This gives an uncontingent estimate for $(\rho, u, B)$ and thereby proving Theorem 2.2

Throughout this section, $C$ will denote a generic positive constant which depends on the same quantities as the constant $C$ in the statement of Theorem 2.2 but independent of time $t$ and the regularity of initial data.

We first recall the following estimates on the effective viscous flux $F$ which is defined in (1.7).

Lemma 2.3. Assume that $\rho$ satisfies (2.4). For each $p>1$, there is a constant $C>0$ such that for all $t>0$, we have

$$
\|F(\cdot, t)\|_{L^{p}} \leq C\left[\|\nabla u(\cdot, t)\|_{L^{p}}+\|(\rho-\tilde{\rho})(\cdot, t)\|_{L^{p}}\right],
$$

and

$$
\|\nabla F(\cdot, t)\|_{L^{p}} \leq C\left[\|\dot{u}(\cdot, t)\|_{L^{p}}+\|B \nabla B(\cdot, t)\|_{L^{p}}\right]
$$

Proof. The assertion (2.6) follows immediately from the definition of $F$, and the proof of (2.7) relies on the Poisson equation (1.8) and the Marcinkiewicz multiplier theorem (refer to Stein [21], pg. 96).

Using the estimates (2.6)-(2.7) on $F$, we have the following estimates on $\nabla u$ and $\nabla \omega:$ 
Lemma 2.4. Assume that $\rho$ satisfies (2.4). For each $p>1$, there is a constant $C>0$ depends on $p$ such that for all $t>0$, we have

$$
\begin{gathered}
\|\nabla u(\cdot, t)\|_{L^{p}} \leq C\left[\|F(\cdot, t)\|_{L^{p}}+\|\omega(\cdot, t)\|_{L^{p}}+\|(P-\tilde{P})(\cdot, t)\|_{L^{p}}\right], \\
\|\nabla \omega(\cdot, t)\|_{L^{p}} \leq C\left[\|\dot{u}(\cdot, t)\|_{L^{p}}+\|B \nabla B(\cdot, t)\|_{L^{p}}\right] .
\end{gathered}
$$

Proof. By the definition (1.7) of $F$,

$$
(\mu+\lambda) \Delta u^{j}=F_{x_{j}}+(\mu+\lambda) \omega_{x_{k}}^{j, k}+(P-\tilde{P})_{x_{j}} .
$$

Hence by differentiating and taking the Fourier transform on the above equation, we can apply Marcinkiewicz multiplier theorem in a similar as we did in Lemma 2.3 and (2.8) follows.

For the case of $\nabla \omega$, by direct computation, we have

$$
\mu \Delta \omega=\left(\rho \dot{u}^{j}\right)_{x_{k}}-\left(\rho \dot{u}^{k}\right)_{x_{j}}-\left(\nabla B^{j} \cdot B\right)_{x_{k}}+\left(\nabla B^{k} \cdot B\right)_{x_{j}},
$$

and using the same argument as for $\nabla u,(2.9)$ immediately follows.

We now start giving the estimates on $(\rho, u, B)$ which will be used in deriving (2.3). We begin with the following $L^{2}$ estimates on $(\rho, u, B)$ for all $T>0$ :

Lemma 2.5. Assume that $\rho$ satisfies (2.4). For $T>0$, we have

$$
\sup _{0 \leq \tau \leq T} \int_{\mathbb{R}^{3}}\left(|\rho-\tilde{\rho}|^{2}+\rho|u|^{2}+|B|^{2}\right) d x+\int_{0}^{T} \int_{\mathbb{R}^{3}}\left(|\nabla u|^{2}+|\nabla B|^{2}\right) d x d \tau \leq C C_{0} .
$$

Proof. The bound (2.10) follows from the standard energy balance equation, namely

$$
\begin{aligned}
\int_{\mathbb{R}^{3}} & \left.\left(\frac{1}{2} \rho|u|^{2}+\mathcal{G}(\rho)\right) d x\right|_{0} ^{t}+\left.\int_{\mathbb{R}^{3}} \frac{1}{2}|B|^{2} d x\right|_{0} ^{t} \\
& +\int_{0}^{t} \int_{\mathbb{R}^{3}}\left(\mu|\nabla u|^{2}+\lambda(\operatorname{div}(u))^{2}+\nu|\nabla B|^{2}\right) d x d \tau=0,
\end{aligned}
$$

where $\int_{\mathbb{R}^{d}} \mathcal{G}(\rho) d x=\int_{\mathbb{R}^{d}}\left(\rho \int_{\tilde{\rho}}^{\rho} \tau^{-2}(P(\tau)-P(\tilde{\rho})) d \tau\right) d x$ is comparable to the $L^{2}\left(\mathbb{R}^{3}\right)$ norm of $(\rho-\tilde{\rho})$ (see [10] for related discussion).

To proceed further, we have to obtain higher order estimates on $u$ and $B$. Due to the intricate coupling effects between $u$ and $B$, we subdivide the estimates into two cases namely $T \leq 1$ and $T>1$. These will be illustrated in subsection 2.1 and subsection 2.2 as follows:

2.1. Estimates on $u$ and $B$ for $T \leq 1$. In this subsection, we obtain estimates on $u$ and $B$ for $T \leq 1$. We start with the following $L^{6}$ estimates on $u$ and $B$ :

Lemma 2.6. Assume that $\rho$ satisfies (2.4). For $T \leq 1$, we have

$$
\sup _{0 \leq \tau \leq T} \int_{\mathbb{R}^{3}}\left(|u|^{6}+|B|^{6}\right) d x \leq C_{L} C_{0}^{\theta_{q}},
$$

where $C_{L}, \theta_{q}>0$ depends on $L$ and $q$ respectively. 
Proof. We follow the computations given in 22] and obtain, for $0 \leq t \leq T \leq 1$,

$$
\begin{aligned}
&\left.\int_{\mathbb{R}^{3}}\left(|u|^{6}+|B|^{6}\right) d x\right|_{\tau=0} ^{t}+6 \int_{0}^{T} \int_{\mathbb{R}^{3}}\left(\mu|u|^{2}|\nabla u|^{2}+\nu|B|^{2}|\nabla B|^{2}\right) d x d \tau \\
&\left.\left.+(-24 \lambda+6 \mu) \int_{0}^{T} \int_{\mathbb{R}^{3}}|u|^{2}\left|\nabla\left(|u|^{2}\right)\right|^{2} d x d \tau+6 \nu \int_{0}^{T} \int_{\mathbb{R}^{3}}|B|^{2} \mid \nabla\right)|B|^{2}\right)\left.\right|^{2} d x d \tau \\
& \leq C {\left[\int_{0}^{T} \int_{\mathbb{R}^{3}}|\rho-\tilde{\rho}|\left|\operatorname{div}\left(|u|^{4} u\right)\right| d x d \tau+\int_{0}^{T} \int_{\mathbb{R}^{3}}|u|^{4}|u|\left|\operatorname{div}\left(B B^{T}\right)\right| d x d \tau\right] } \\
&+C\left[\int_{0}^{T} \int_{\mathbb{R}^{3}}|u|^{4}|u|\left|\nabla\left(\frac{1}{2}|B|^{2}\right)\right| d x d \tau+\int_{0}^{T} \int_{\mathbb{R}^{3}}|B|^{4}\left|B \cdot \operatorname{div}\left(B u^{T}-u B^{T}\right)\right| d x d \tau\right] .
\end{aligned}
$$

By the assumption (1.9), the term involving $(-24 \lambda+6 \mu)$ is positive, while the term $C \int_{\mathbb{R}^{3}}\left(\left|u_{0}\right|^{6}+\left|B_{0}\right|^{6}\right) d x$ can be bounded in terms of $C_{0}$ and $L$ by interpolation and assumption (1.18). The rest of the analysis follows by a Gronwäll-type argument (also see 22 for details) and we omit the details here.

Next we derive bounds for $u$ and $B$ in $L^{\infty}\left([0, T] ; H^{1}\left(\mathbb{R}^{3}\right)\right)$ when $T \leq 1$.

Lemma 2.7. Assume that $\rho$ satisfies (2.4) and $C_{0} \ll 1$. For $T \leq 1$ and $s \in[0,1]$, we have

$$
\sup _{0 \leq \tau \leq T} \tau^{1-s} \int_{\mathbb{R}^{3}}\left(|\nabla u|^{2}+|\nabla B|^{2}\right) d x+\int_{\mathbb{R}^{3}} \tau^{1-s}\left(|\dot{u}|^{2}+\left|B_{t}\right|^{2}\right) d x \leq C C_{0} .
$$

Proof. We apply the interpolation argument as given in Hoff [11. We define differential operators $\mathcal{L}_{u}, \mathcal{L}_{B}$ acting on functions $w: \mathbb{R}^{3} \times[0, \infty) \rightarrow \mathbb{R}^{3}$ by

$$
\begin{aligned}
& \left(\mathcal{L}_{u} w\right)^{j}=\left(\rho w^{j}\right)_{t}+\operatorname{div}\left(\rho w^{j} u\right)+\left(\frac{1}{2}|B|^{2}\right)_{x_{j}}-\operatorname{div}\left(B^{j} B\right)-\left(\mu \Delta u^{j}+\lambda \operatorname{div} u_{x_{j}}\right), \\
& \left(\mathcal{L}_{B} w\right)^{j}=w_{t}^{j}+\operatorname{div}\left(w^{j} u-u^{j} w\right)-\nu \Delta w^{j} .
\end{aligned}
$$

Then we define $w_{1}$ and $w_{2}$ by

$$
\mathcal{L}_{u} w_{1}=0, w_{1}(x, 0)=w_{10}(x), \quad \mathcal{L}_{u} w_{2}=-\nabla P(\rho), w_{2}(x, 0)=0,
$$

for a given $w_{10}$. Notice that $\mathcal{L}_{B} B=0$, and if $w_{10}=u_{0}$, then $w_{1}+w_{2}=u$. Using the energy estimate as obtained in Lemma 2.5, we have

$$
\begin{aligned}
& \sup _{0 \leq \tau \leq T} \int_{\mathbb{R}^{3}}\left(\left|w_{1}(x, t)\right|^{2}+|B|^{2}\right) d x+\int_{0}^{T} \int_{\mathbb{R}^{3}}\left(\left|\nabla w_{1}\right|^{2}+|\nabla B|^{2}\right) d x d \tau \\
& \quad \leq C \int_{\mathbb{R}^{3}}\left(\left|w_{10}\right|^{2}+\left|B_{0}\right|^{2}\right) d x,
\end{aligned}
$$

as well as

$$
\begin{gathered}
\sup _{0 \leq \tau \leq T} \int_{\mathbb{R}^{3}}\left(\left|w_{2}(x, \tau)\right|^{2}+|B|^{2}\right) d x+\int_{0}^{T} \int_{\mathbb{R}^{3}}\left(\left|\nabla w_{2}\right|^{2}+|\nabla B|^{2}\right) d x d \tau \\
\leq C \int_{\mathbb{R}^{3}}\left|B_{0}\right|^{2} d x+C T \sup _{0 \leq \tau \leq T}\|(P-\tilde{P})(\cdot, \tau)\|_{L^{2}}^{2},
\end{gathered}
$$


Also, for $k \in\{0,1\}$ and $0 \leq t \leq T$, we have

$$
\begin{aligned}
& \left.\tau^{k} \int_{\mathbb{R}^{3}}\left(\left|\nabla w_{1}(x, \tau)\right|^{2}+|\nabla B|^{2}\right) d x\right|_{\tau=0} ^{\tau=t}+\int_{0}^{T} \int_{\mathbb{R}^{3}} \tau^{k}\left(\rho\left|\dot{w}_{1}\right|^{2}+\left|B_{t}\right|^{2}\right) d x d \tau \\
& \leq C\left(\int_{0}^{T} \int_{\mathbb{R}^{3}} k \tau^{k-1}\left(\left|\nabla w_{1}\right|^{2}+|\nabla B|^{2}\right) d x d \tau+\int_{0}^{T} \int_{\mathbb{R}^{3}} \tau^{\frac{3 k}{2}}\left(\left|\nabla w_{1}\right|^{3}+|\nabla B|^{3}\right) d x d \tau\right. \\
& \quad+C \int_{0}^{T} \int_{\mathbb{R}^{3}} \tau^{k}\left(|\nabla B|^{2}|B|^{2}+|\nabla B|^{2}|u|^{2}+|\nabla u|^{2}|B|^{2}\right) d x d \tau
\end{aligned}
$$

and

$$
\begin{aligned}
& \left.\int_{\mathbb{R}^{3}}\left|\nabla w_{2}(x, \tau)\right|^{2} d x\right|_{\tau=0} ^{\tau=t}+\int_{0}^{T} \int_{\mathbb{R}^{3}} \rho\left|\dot{w}_{2}\right|^{2} d x d \tau \\
& \leq C\left(\left|\int_{\mathbb{R}^{3}}(P-\tilde{P}) \operatorname{div}\left(w_{2}\right)(x, \tau) d x\right|_{\tau=0}^{\tau=t} \mid\right) \\
& \quad+C\left(\int_{0}^{T} \int_{\mathbb{R}^{3}} \tau^{\frac{3 k}{2}}\left(\left|\nabla w_{2}\right|^{3}+|\nabla B|^{3}\right) d x d \tau+\int_{0}^{T} \int_{\mathbb{R}^{3}} \tau^{k}|\nabla B|^{2}|B|^{2} d x d \tau\right) .
\end{aligned}
$$

Using (2.13)-(2.14), the terms

$$
\int_{0}^{T} \int_{\mathbb{R}^{3}} k \tau^{k-1}\left(\left|\nabla w_{1}\right|^{2}+|\nabla B|^{2}\right) d x d \tau
$$

and

$$
\left|\int_{\mathbb{R}^{3}}(P-\tilde{P}) \operatorname{div}\left(w_{2}\right)(x, \tau) d x\right|_{\tau=0}^{\tau=t} \mid
$$

can be bounded in terms of $C_{0}$, namely

$$
\left|\int_{0}^{T} \int_{\mathbb{R}^{3}} k \tau^{k-1}\left(\left|\nabla w_{1}\right|^{2}+|\nabla B|^{2}\right) d x d \tau\right| \leq C \int_{0}^{T} \int_{\mathbb{R}^{3}}\left(\left|\nabla w_{1}\right|^{2}+|\nabla B|^{2}\right) d x d \tau \leq C C_{0}
$$

and

$$
\begin{aligned}
& \left|\int_{\mathbb{R}^{3}}(P-\tilde{P}) \operatorname{div}\left(w_{2}\right)(x, \tau) d x\right|_{\tau=0}^{\tau=t} \mid \\
& \leq C\left(\int_{\mathbb{R}^{3}}|\rho-\tilde{\rho}|^{2}(x, t) d x\right)^{\frac{1}{2}}\left(\int_{\mathbb{R}^{3}}\left|\nabla w_{2}\right|^{2}(x, t) d x\right)^{\frac{1}{2}} \leq C C_{0}^{\frac{1}{2}}\left(\int_{\mathbb{R}^{3}}\left|\nabla w_{2}\right|^{2}(x, t) d x\right)^{\frac{1}{2}} .
\end{aligned}
$$

We now aim at controlling the higher terms as appeared on the right sides of (2.15) and (2.16). In view of the bound (2.11), it suffices to consider

$$
\int_{0}^{T} \int_{\mathbb{R}^{3}} \tau^{\frac{3 k}{2}}\left(\left|\nabla w_{i}\right|^{3}+|\nabla u|^{3}+|\nabla B|^{3}\right) d x d \tau
$$


for $i=1,2$. For the term involving $\nabla B$, using (1.14), we can estimate it as follows.

$$
\begin{aligned}
& \int_{0}^{t} \int_{\mathbb{R}^{3}} \tau^{\frac{3 k}{2}}|\nabla B|^{3} d x d \tau \\
& \leq C \int_{0}^{t} \int_{\mathbb{R}^{3}} \tau^{\frac{3 k}{2}}\left(\int_{\mathbb{R}^{3}}|\Delta B|^{2}\right)^{\frac{3}{4}}\left(\int_{\mathbb{R}^{3}}|\nabla B|^{2}\right)^{\frac{3}{4}} d x d \tau \\
& \leq C \int_{0}^{t} \tau^{\frac{3 k}{2}}\left(\int_{\mathbb{R}^{3}}\left(\left|B_{t}\right|^{2}+|\nabla B|^{2}|u|^{2}+|\nabla u|^{2}|B|^{2}\right)\right)^{\frac{3}{4}}\left(\int_{\mathbb{R}^{3}}|\nabla B|^{2}\right)^{\frac{3}{4}} \\
& \leq C\left(\sup _{0 \leq \tau \leq T} \tau^{k} \int_{\mathbb{R}^{3}}|\nabla B|^{2} d x\right)^{\frac{1}{2}}\left(\int_{0}^{t} \int_{\mathbb{R}^{3}} \tau^{k}\left|B_{t}\right|^{2} d x\right)^{\frac{3}{4}}\left(\int_{0}^{t} \int_{\mathbb{R}^{3}}|\nabla B|^{2} d x\right)^{\frac{1}{4}} d \tau \\
& \quad+C \int_{0}^{t} \tau^{\frac{3 k}{2}}\left(\int_{\mathbb{R}^{3}}|\nabla B|^{3} d x\right)^{\frac{1}{2}}\left(\int_{\mathbb{R}^{3}}|u|^{6} d x\right)^{\frac{1}{4}}\left(\int_{\mathbb{R}^{3}}|\nabla B|^{2} d x\right)^{\frac{3}{4}} d \tau \\
& \quad+C \int_{0}^{t} \tau^{\frac{3 k}{2}}\left(\int_{\mathbb{R}^{3}}|\nabla u|^{3} d x\right)^{\frac{1}{2}}\left(\int_{\mathbb{R}^{3}}|B|^{6} d x\right)^{\frac{1}{4}}\left(\int_{\mathbb{R}^{3}}|\nabla B|^{2} d x\right)^{\frac{3}{4}} d \tau .
\end{aligned}
$$

Therefore, by the bounds (2.10) and (2.11), we obtain

$$
\begin{aligned}
& \int_{0}^{t} \int_{\mathbb{R}^{3}} \tau^{\frac{3 k}{2}}|\nabla B|^{3} d x d \tau \\
& \leq C C_{0}^{\frac{1}{4}}\left(\sup _{0 \leq \tau \leq T} \tau^{k} \int_{\mathbb{R}^{3}}|\nabla B|^{2} d x\right)^{\frac{1}{2}}\left(\int_{0}^{t} \int_{\mathbb{R}^{3}} \tau^{k}\left|B_{t}\right|^{2} d x d \tau\right)^{\frac{3}{4}} \\
& \quad+C C_{0}^{\frac{1}{4}} C_{0}^{\frac{1}{2}}\left(\sup _{0 \leq \tau \leq T} \tau^{k} \int_{\mathbb{R}^{3}}|\nabla B|^{2} d x\right)^{\frac{1}{4}}\left(\int_{0}^{t} \int_{\mathbb{R}^{3}} \tau^{\frac{3 k}{2}}|\nabla B|^{3} d x d \tau\right)^{\frac{1}{2}} \\
& \quad+C C_{0}^{\frac{1}{4}} C_{0}^{\frac{1}{2}}\left(\sup _{0 \leq \tau \leq T} \tau^{k} \int_{\mathbb{R}^{3}}|\nabla B|^{2} d x\right)^{\frac{1}{4}}\left(\int_{0}^{t} \int_{\mathbb{R}^{3}} \tau^{\frac{3 k}{2}}|\nabla u|^{3} d x d \tau\right)^{\frac{1}{2}} .
\end{aligned}
$$

To estimate the term $\int_{0}^{T} \int_{\mathbb{R}^{3}} t^{\frac{3 k}{2}}|\nabla u|^{3}$, we apply (1.14) and the bounds (2.7)-(2.8) to obtain

$$
\begin{aligned}
& \int_{0}^{t} \int_{\mathbb{R}^{3}} \tau^{\frac{3 k}{2}}|\nabla u|^{3} d x d \tau \\
& \leq C \int_{0}^{t} \int_{\mathbb{R}^{3}} \tau^{\frac{3 k}{2}}\left(|F|^{3}+|\omega|^{3}+|P-\tilde{P}|^{3}\right) d x d \tau \\
& \leq C \int_{0}^{t} \tau^{\frac{3 k}{2}}\left(\int_{\mathbb{R}^{3}}|F|^{2} d x\right)^{\frac{3}{4}}\left(\int_{\mathbb{R}^{3}}|\nabla F|^{2} d x\right)^{\frac{3}{4}} d \tau+C \int_{0}^{t} \int_{\mathbb{R}^{3}} \tau^{\frac{3 k}{2}}\left(|\omega|^{3}+|P-\tilde{P}|^{3}\right) d x d \tau \\
& \leq C C_{0}^{\frac{1}{4}}\left(\sup _{0 \leq \tau \leq T} \tau^{k} \int_{\mathbb{R}^{3}}|\nabla u|^{2} d x\right)^{\frac{1}{2}}\left(\int_{0}^{t} \int_{\mathbb{R}^{3}} \tau^{k}|\dot{u}|^{2} d x d \tau\right)^{\frac{3}{4}}+C_{0}^{\frac{3}{4}}\left(\int_{0}^{t} \int_{\mathbb{R}^{3}} \tau^{k}|\dot{u}|^{2} d x d \tau\right)^{\frac{3}{4}} \\
& \quad+C C_{0}^{\frac{1}{4}} C_{0}^{\frac{1}{2}}\left(\sup _{0 \leq \tau \leq T} \tau^{k} \int_{\mathbb{R}^{3}}|\nabla u|^{2} d x\right)^{\frac{1}{4}}\left(\int_{0}^{t} \int_{\mathbb{R}^{3}} \tau^{\frac{3 k}{2}}|\nabla B|^{3} d x d \tau\right)^{\frac{1}{2}} \\
& \quad+C C_{0}\left(\int_{0}^{t} \int_{\mathbb{R}^{3}} \tau^{\frac{3 k}{2}}|\nabla B|^{3} d x d \tau\right)^{\frac{1}{2}}+C \int_{0}^{t} \int_{\mathbb{R}^{3}} \tau^{\frac{3 k}{2}}\left(|\omega|^{3}+|P-\tilde{P}|^{3}\right) d x d \tau .
\end{aligned}
$$

The estimates for $w_{1}$ and $w_{2}$ are just similar. In view of the above, under suitable smallness assumption on the initial data, the integrals $\int_{0}^{T} \int_{\mathbb{R}^{3}} \tau^{\frac{3 k}{2}}\left(|\nabla u|^{3}+|\nabla B|^{3}\right)$ can be absorbed by the left sides of (2.15) and (2.16). We treat $w_{1}$ and $w_{2}$ in a 
similar way and obtain the following estimates on $w_{1}, w_{2}$ and $B$ :

$$
\begin{gathered}
\sup _{0 \leq \tau \leq T} \int_{\mathbb{R}^{3}}\left(\left|\nabla w_{1}\right|^{2}+|\nabla B|^{2}\right) d x+\int_{0}^{T} \int_{\mathbb{R}^{3}}\left(\left|\dot{w}_{1}\right|^{2}+\left|B_{t}\right|^{2}\right) d x d \tau \\
\leq C\left(\left\|w_{10}\right\|_{H^{1}}^{2}+\left\|B_{0}\right\|_{H^{1}}^{2}\right), \\
\sup _{0 \leq \tau \leq T} t \int_{\mathbb{R}^{3}}\left(\left|\nabla w_{1}\right|^{2}+|\nabla B|^{2}\right) d x+\int_{0}^{T} \int_{\mathbb{R}^{3}} \tau\left(\left|\dot{w}_{1}\right|^{2}+\left|B_{t}\right|^{2}\right) d x d \tau \\
\leq C\left(\left\|w_{10}\right\|_{L^{2}}^{2}+\left\|B_{0}\right\|_{L^{2}}^{2}\right), \\
\sup _{0 \leq \tau \leq T} \int_{\mathbb{R}^{3}}\left|\nabla w_{2}\right|^{2} d x+\int_{0}^{T} \int_{\mathbb{R}^{3}}\left|\dot{w}_{2}\right|^{2} d x d \tau \leq C C_{0} .
\end{gathered}
$$

Since the operators $\mathcal{L}_{u}$ and $\mathcal{L}_{B}$ are both linear, we can apply Riesz-Thorin interpolation to deduce from (2.17)-(2.18) that

$$
\begin{aligned}
& \sup _{0 \leq t \leq 1} \tau^{1-s} \int_{\mathbb{R}^{3}}\left(\left|\nabla w_{1}\right|^{2}+|\nabla B|^{2}\right) d x+\int_{0}^{T} \int_{\mathbb{R}^{3}} \tau^{1-s}\left(\left|\dot{w}_{1}\right|^{2}+\left|B_{t}\right|^{2}\right) d x d \tau \\
& \leq C\left(\left\|w_{10}\right\|_{H^{s}}^{2}+\left\|B_{0}\right\|_{H^{s}}^{2}\right) .
\end{aligned}
$$

By taking $w_{10}=u_{0}$, we conclude from (2.19) and (2.20) that

$$
\begin{aligned}
\sup _{0 \leq \tau \leq T} \tau^{1-s} \int_{\mathbb{R}^{3}}\left(|\nabla u|^{2}+|\nabla B|^{2}\right) d x \\
\quad+\int_{0}^{T} \int_{\mathbb{R}^{3}} \tau^{1-s}\left(|\dot{u}|^{2}+\left|B_{t}\right|^{2}\right) d x d \tau \leq C C_{0} .
\end{aligned}
$$

Next we give the following auxiliary bounds which are useful in estimating some mixed terms in $u$ and $B$ when $T \leq 1$.

Lemma 2.8. Assume that $\rho$ satisfies (2.4) and $C_{0} \ll 1$. For $T \leq 1$ and $s \in[0,1]$, we have

$$
\int_{0}^{T} \int_{\mathbb{R}^{3}} \tau^{1-s}\left(|\nabla B|^{2}|B|^{2}+|\nabla u|^{2}|B|^{2}+|\nabla B|^{2}|u|^{2}\right) d x d \tau \leq C C_{0}^{\theta}
$$

for some $\theta>0$.

Proof. Using equation (1.3) and the bounds (2.10)-(2.11) and (2.12), we have

$$
\begin{aligned}
& \int_{0}^{T} \tau^{1-s} \int_{\mathbb{R}^{3}}|\nabla B|^{2}|B|^{2} d x d \tau \\
& \leq \int_{0}^{T} \tau^{1-s}\left(\int_{\mathbb{R}^{3}}|\nabla B|^{3} d x\right)^{\frac{2}{3}}\left(\int_{\mathbb{R}^{3}}|B|^{6} d x\right)^{\frac{1}{3}} d \tau \\
& \leq C \int_{0}^{T} \tau^{1-s}\left(\int_{\mathbb{R}^{3}}|\nabla B|^{2} d x\right)^{\frac{1}{2}}\left(\int_{\mathbb{R}^{3}}|\Delta B|^{2} d x\right)^{\frac{1}{2}}\left(\int_{\mathbb{R}^{3}}|B|^{6} d x\right)^{\frac{1}{3}} d \tau \\
& \leq C \int_{0}^{T} \tau^{1-s}\left(\int_{\mathbb{R}^{3}}|\nabla B|^{2} d x\right)^{\frac{1}{2}}\left(\int_{\mathbb{R}^{3}}\left(|B|^{2}+|\nabla u|^{2}|B|^{2}+|\nabla B|^{2}|u|^{2}\right) d x\right)^{\frac{1}{2}}\left(\int_{\mathbb{R}^{3}}|B|^{6} d x\right)^{\frac{1}{3}} d \tau \\
& \leq C C_{0}^{\frac{1}{3}} C_{0}^{\frac{1}{2}}\left(C_{0}+\int_{0}^{T} \int_{\mathbb{R}^{3}} \tau^{1-s}\left(|\nabla u|^{2}|B|^{2}+|\nabla B|^{2}|u|^{2}\right) d x d \tau\right)^{\frac{1}{2}} \cdot
\end{aligned}
$$


Similarly, we have

$$
\begin{aligned}
& \int_{0}^{T} \tau^{1-s} \int_{\mathbb{R}^{3}}|\nabla B|^{2}|u|^{2} d x d \tau \\
& \leq C C_{0}^{\frac{1}{3}} C_{0}^{\frac{1}{2}}\left(C_{0}+\int_{0}^{T} \int_{\mathbb{R}^{3}} \tau^{1-s}\left(|\nabla u|^{2}|B|^{2}+|\nabla B|^{2}|u|^{2}\right) d x d \tau\right)^{\frac{1}{2}} .
\end{aligned}
$$

Finally, for the term $\int_{0}^{T} \int_{\mathbb{R}^{3}} \tau^{1-s}|\nabla u|^{2}|B|^{2}$, using the bound (2.8) on $\nabla u$,

$$
\begin{aligned}
& \int_{0}^{T} \int_{\mathbb{R}^{3}} \tau^{1-s}|\nabla u|^{2}|B|^{2} d x d \tau \\
& \leq C \int_{0}^{T} \tau^{1-s}\left(\int_{\mathbb{R}^{3}}|\nabla u|^{3} d x\right)^{\frac{2}{3}}\left(\int_{\mathbb{R}^{3}}|B|^{6} d x\right)^{\frac{1}{3}} d \tau \\
& \leq C C_{0}^{\frac{1}{3}} \int_{0}^{T} \tau^{1-s}\left(\int_{\mathbb{R}^{3}}\left(|F|^{3}+|\omega|^{3}+|\rho-\tilde{\rho}|^{3}\right) d x\right)^{\frac{2}{3}} d \tau .
\end{aligned}
$$

To bound the term involving $F$ in (2.22), using the estimates (2.6)-(2.7), we have

$$
\begin{aligned}
& \int_{0}^{T}\left(\int_{\mathbb{R}^{3}}|F|^{3} d x\right)^{\frac{2}{3}} d \tau \\
& \leq C \int_{0}^{T} \tau^{1-s}\left(\int_{\mathbb{R}^{3}}|F|^{2} d x\right)^{\frac{1}{2}}\left(\int_{\mathbb{R}^{3}}|\nabla F|^{2} d x\right)^{\frac{1}{2}} d \tau \\
& \leq C \int_{0}^{T} \tau^{1-s}\left(\int_{\mathbb{R}^{3}}\left(|\nabla u|^{2}+|P-\tilde{P}|^{2}\right) d x\right)^{\frac{1}{2}}\left(\int_{\mathbb{R}^{3}}\left(|\dot{u}|^{2}+|B|^{2}|\nabla B|^{2}\right) d x\right)^{\frac{1}{2}} d \tau \\
& \leq C C_{0}^{\frac{1}{2}}\left(C_{0}+\int_{0}^{T} \tau^{1-s} \int_{\mathbb{R}^{3}}|B|^{2}|\nabla B|^{2} d x d \tau\right)^{\frac{1}{2}} .
\end{aligned}
$$

The other terms on the right side of (2.22) can be treated in a similar way, so we conclude that

$$
\begin{aligned}
& \int_{0}^{T} \int_{\mathbb{R}^{3}} \tau^{1-s}\left(|\nabla B|^{2}|B|^{2}+|\nabla u|^{2}|B|^{2}+|\nabla B|^{2}|u|^{2}\right) d x d \tau \\
& \quad \leq C C_{0}^{\frac{1}{3}} C_{0}^{\frac{1}{2}}\left(C_{0}+\int_{0}^{T} \int_{\mathbb{R}^{3}} \tau^{1-s}\left(|\nabla B|^{2}|B|^{2}+|\nabla u|^{2}|B|^{2}+|\nabla B|^{2}|u|^{2}\right) d x d \tau\right)^{\frac{1}{2}}
\end{aligned}
$$

and (2.21) follows.

We now derive preliminary bounds for $\dot{u}$ and $B_{t}$ in $L^{\infty}\left([0, T] ; L^{2}\left(\mathbb{R}^{3}\right)\right)$ when $T \leq 1$. We point out that here we require $s \in\left(\frac{1}{2}, 1\right]$ on the time layer factor $\tau^{2-s}$ due to the lack of integrability in time near $t=0$ for $\dot{u}$ and $B_{t}$.

Lemma 2.9. Assume that $\rho$ satisfies (2.4) and $C_{0} \ll 1$. For $T \leq 1$ and $s \in\left(\frac{1}{2}, 1\right]$, we have

$$
\sup _{0 \leq \tau \leq T} \tau^{2-s} \int_{\mathbb{R}^{3}}\left(|\dot{u}|^{2}+\left|B_{t}\right|^{2}\right) d x+\int_{0}^{T} \int_{\mathbb{R}^{3}} \tau^{2-s}\left(|\nabla \dot{u}|^{2}+\left|\nabla B_{t}\right|^{2}\right) d x d \tau \leq C C_{0}^{\theta},
$$

for some $\theta>0$. 
Proof. Following the steps given in 22, we arrive at

$$
\begin{aligned}
& \sup _{0 \leq \tau \leq T} \tau^{2-s} \int_{\mathbb{R}^{3}}\left(|\dot{u}|^{2}+\left|B_{t}\right|^{2}\right) d x+\int_{0}^{T} \int_{\mathbb{R}^{3}} \tau^{2-s}\left(|\nabla \dot{u}|^{2}+\left|\nabla B_{t}\right|^{2}\right) d x d \tau \\
& \leq C C_{0}+C \int_{0}^{T} \int_{\mathbb{R}^{3}} \tau^{2-s}|B|^{2}|u|^{2}\left(|\nabla u|^{2}+|\nabla B|^{2}\right) d x d \tau \\
& \quad+C \int_{0}^{T} \int_{\mathbb{R}^{3}} \tau^{2-s}\left(|B|^{2}\left|B_{t}\right|^{2}+|u|^{2}|\dot{u}|^{2}+\left|B_{t}\right|^{2}|u|^{2}\right) d x d \tau \\
& \quad+C \int_{0}^{T} \int_{\mathbb{R}^{3}} \tau^{2-s}\left(|\nabla u|^{4}+|\nabla B|^{4}\right) d x d \tau .
\end{aligned}
$$

To facilitate the proof, we define the following auxiliary functionals:

$$
\begin{aligned}
& \mathcal{A}_{1}(T)=\sup _{0 \leq \tau \leq T} \tau^{2-s} \int_{\mathbb{R}^{3}}\left(|\dot{u}|^{2}+\left|B_{t}\right|^{2}\right) d x+\int_{0}^{T} \int_{\mathbb{R}^{3}} \tau^{2-s}\left(|\nabla \dot{u}|^{2}+\left|\nabla B_{t}\right|^{2}\right) d x d \tau, \\
& \mathcal{A}_{2}(T)=\int_{0}^{T} \int_{\mathbb{R}^{3}} \tau^{2-s}\left(|\nabla u|^{4}+|\nabla B|^{4}\right) d x d \tau, \\
& \mathcal{A}_{3}(T)=\sup _{0 \leq \tau \leq T} \int_{\mathbb{R}^{3}} \tau^{2-s}\left(|\nabla B|^{2}|B|^{2}+|\nabla u|^{2}|B|^{2}+|\nabla B|^{2}|u|^{2}\right) d x .
\end{aligned}
$$

Our goal is to prove that

$$
\mathcal{A}_{1}+\mathcal{A}_{2}+\mathcal{A}_{3} \leq C C_{0}^{\theta},
$$

for some $\theta>0$. We first consider the right side of (2.24). To bound the term $\int_{0}^{T} \int_{\mathbb{R}^{3}} \tau^{2-s}|B|^{2}\left|B_{t}\right|^{2} d x d \tau$, using the bound (2.12), we have

$$
\begin{aligned}
& \int_{0}^{T} \int_{\mathbb{R}^{3}} \tau^{2-s}|B|^{2}\left|B_{t}\right|^{2} d x d \tau \\
& \leq\left(\int_{0}^{T} \int_{\mathbb{R}^{3}}|B|^{6} d x d \tau\right)^{\frac{1}{3}}\left(\int_{0}^{T} \int_{\mathbb{R}^{3}} t^{\frac{6-3 s}{2}}\left|B_{t}\right|^{2} d x d \tau\right)^{\frac{2}{3}} \\
& \leq C C_{0}^{\frac{1}{3}}\left(\sup _{0 \leq \tau \leq T} \tau^{2-s} \int_{\mathbb{R}^{3}}\left|B_{t}\right|^{2} d x\right)^{\frac{1}{3}}\left(\int_{0}^{T} \int_{\mathbb{R}^{3}} \tau^{2-s}\left|B_{t}\right|^{2} d x d \tau\right)^{\frac{1}{6}} \\
& \quad \times\left(\int_{0}^{T} \int_{\mathbb{R}^{3}} \tau^{2-s}\left|\nabla B_{t}\right|^{2} d x d \tau\right)^{\frac{1}{2}} \\
& \leq C C_{0}^{\frac{1}{3}} \mathcal{A}_{1}^{\frac{1}{3}} C_{0}^{\frac{1}{6}} \mathcal{A}_{1}^{\frac{1}{2}}=C C_{0}^{\frac{1}{2}} \mathcal{A}_{1}^{\frac{5}{6}} .
\end{aligned}
$$

Similarly, the term $\int_{0}^{T} \int_{\mathbb{R}^{3}} \tau^{2-s}|B|^{2}|\dot{u}|^{2}$ can be bounded by

$$
\begin{aligned}
& \int_{0}^{T} \int_{\mathbb{R}^{3}} \tau^{2-s}|B|^{2}|\dot{u}|^{2} d x d \tau \\
& \leq C\left(\int_{0}^{T} \int_{\mathbb{R}^{3}}|B|^{6} d x d \tau\right)^{\frac{1}{3}}\left(\sup _{0 \leq \tau \leq T} \tau^{2-s} \int_{\mathbb{R}^{3}}|\dot{u}|^{2} d x\right)^{\frac{1}{3}} \\
& \quad \times\left(\int_{0}^{T} \int_{\mathbb{R}^{3}} \tau^{2-s}|\dot{u}|^{2} d x d \tau\right)^{\frac{1}{6}}\left(\int_{0}^{T} \int_{\mathbb{R}^{3}} \tau^{2-s}|\nabla \dot{u}|^{2} d x d \tau\right)^{\frac{1}{2}} \\
& \leq C C_{0}^{\frac{1}{2}} \mathcal{A}_{1}^{\frac{5}{6}} .
\end{aligned}
$$


To bound the term $\int_{0}^{T} \int_{\mathbb{R}^{3}} \tau^{2-s}|B|^{2}|u|^{2}\left(|\nabla u|^{2}+|\nabla B|^{2}\right) d x d \tau$, we have

$$
\begin{aligned}
& \int_{0}^{T} \int_{\mathbb{R}^{3}} \tau^{2-s}|B|^{2}|u|^{2}\left(|\nabla u|^{2}+|\nabla B|^{2}\right) d x d \tau \\
& \leq \int_{0}^{T} \int_{\mathbb{R}^{3}} \tau^{2-s}\left(|\nabla u|^{4}+|\nabla B|^{4}\right) d x d \tau+\int_{0}^{T} \int_{\mathbb{R}^{3}} \tau^{2-s}\left(|B|^{8}+|u|^{8}\right) d x d \tau \\
& \leq \mathcal{A}_{2}+\int_{0}^{T} \int_{\mathbb{R}^{3}} \tau^{2-s}\left(|B|^{8}+|u|^{8}\right) d x d \tau .
\end{aligned}
$$

Using the bounds (1.14) and (1.16), the term $\int_{0}^{T} \int_{\mathbb{R}^{3}} \tau^{2-s}|u|^{8} d x d \tau$ can be estimated as follows.

$$
\begin{aligned}
& \int_{0}^{T} \int_{\mathbb{R}^{3}} \tau^{2-s}|u|^{8} d x d \tau \\
& \leq\left(\sup _{0 \leq \tau \leq T} \int_{\mathbb{R}^{3}}|u|^{4} d x\right)\left(\int_{0}^{T} \tau^{2-s}\|u(\cdot, \tau)\|_{L^{\infty}}^{4} d \tau\right) \\
& \leq C\left(\sup _{0 \leq \tau \leq T} \int_{\mathbb{R}^{3}}|u|^{2} d x\right)^{\frac{1}{2}}\left(\sup _{0 \leq \tau \leq T} \int_{\mathbb{R}^{3}}|u|^{6} d x\right)^{\frac{1}{2}} \\
& \quad \times \int_{0}^{T} \tau^{2-s}\left(\int_{\mathbb{R}^{3}}\left(|u|^{4}+|\nabla u|^{4}\right) d x\right) d \tau \\
& \leq C C_{0}\left[\int_{0}^{T} \tau^{2-s}\left(\int_{\mathbb{R}^{3}}|u|^{2} d x\right)^{\frac{1}{2}}\left(\int_{\mathbb{R}^{3}}|\nabla u|^{2} d x\right)^{\frac{3}{2}} d \tau+\mathcal{A}_{2}\right] \\
& \leq C C_{0}\left(C_{0}^{2}+\mathcal{A}_{2}\right) .
\end{aligned}
$$

The term $\int_{0}^{T} \int_{\mathbb{R}^{3}} \tau^{2-s}|B|^{8} d x d \tau$ can be estimated in a similar way and we obtain

$$
\int_{0}^{T} \int_{\mathbb{R}^{3}} \tau^{2-s}|B|^{2}|u|^{2}\left(|\nabla u|^{2}+|\nabla B|^{2}\right) d x d \tau \leq \mathcal{A}_{2}+C C_{0}\left(C_{0}^{2}+\mathcal{A}_{2}\right)
$$

Therefore we have

$$
\mathcal{A}_{1} \leq C C_{0}^{\frac{1}{2}} \mathcal{A}_{1}^{\frac{5}{6}}+C C_{0}\left(C_{0}^{2}+\mathcal{A}_{2}\right)
$$

Next we consider the term $\mathcal{A}_{2}$. Using the estimate (2.8), we obtain

$$
\int_{0}^{T} \int_{\mathbb{R}^{3}} \tau^{2-s}|\nabla u|^{4} d x d \tau \leq C \int_{0}^{T} \int_{\mathbb{R}^{3}} \tau^{2-s}\left(|F|^{4}+|\omega|^{4}+|P-\tilde{P}|^{4}\right) d x d \tau
$$


For $s \in\left(\frac{1}{2}, 1\right]$, using the bounds (2.12) and (2.21), we have

$$
\begin{aligned}
& \int_{0}^{T} \int_{\mathbb{R}^{3}} \tau^{2-s}|F|^{4} d x d \tau \\
& \leq C\left(\sup _{0 \leq \tau \leq T} \tau^{1-s} \int_{\mathbb{R}^{3}}|F|^{2} d x\right)^{\frac{1}{2}} \\
& \quad \times\left(\sup _{0 \leq \tau \leq T} \tau^{2-s} \int_{\mathbb{R}^{3}}|\nabla F|^{2} d x\right)^{\frac{1}{2}}\left(\int_{0}^{T} \int_{\mathbb{R}^{3}} \tau^{1-s}|\nabla F|^{2} d x d \tau\right) \\
& \leq C C_{0}^{\frac{1}{2}}\left(\sup _{0 \leq \tau \leq T} \tau^{2-s} \int_{\mathbb{R}^{3}}\left(|\dot{u}|^{2}+|\nabla B|^{2}|B|^{2}\right) d x\right)^{\frac{1}{2}} \\
& \quad \times\left(\int_{0}^{T} \int_{\mathbb{R}^{3}} \tau^{1-s}\left(|\dot{u}|^{2}+|\nabla B|^{2}|B|^{2}\right) d x d \tau\right)^{\frac{1}{2}} \\
& \leq C C_{0}^{\frac{1}{2}}\left(\mathcal{A}_{1}+\mathcal{A}_{3}\right)\left(C_{0}+C_{0}^{\theta}\right) .
\end{aligned}
$$

The term $\omega$ and $P-\tilde{P}$ can be treated in a similar way, and we have

$$
\int_{0}^{T} \int_{\mathbb{R}^{3}} \tau^{2-s}|\nabla u|^{4} d x d \tau \leq C C_{0}^{\frac{1}{2}}\left(\mathcal{A}_{1}+\mathcal{A}_{3}\right)\left(C_{0}+C_{0}^{\theta}\right)+C C_{0}
$$

For $\int_{0}^{T} \int_{\mathbb{R}^{3}} \tau^{2-s}|\nabla B|^{4} d x d \tau$, we estimate it as follows.

$$
\begin{aligned}
& \int_{0}^{T} \int_{\mathbb{R}^{3}} \tau^{2-s}|\nabla B|^{4} d x d \tau \\
& \leq C \int_{0}^{T} \tau^{2-s}\left(\int_{\mathbb{R}^{3}}|\nabla B|^{2} d x\right)^{\frac{1}{2}}\left(\int_{\mathbb{R}^{3}}|\Delta B|^{2} d x\right)^{\frac{3}{2}} d \tau \\
& \leq C\left(\sup _{0 \leq \tau \leq T} \tau^{2-s} \int_{\mathbb{R}^{3}}|\Delta B|^{2} d x\right)^{\frac{1}{2}}\left(\sup _{0 \leq \tau \leq T} \tau^{1-s} \int_{\mathbb{R}^{3}}|\nabla B|^{2} d x\right)^{\frac{1}{2}} \\
& \quad \times\left(\int_{0}^{T} \int_{\mathbb{R}^{3}} \tau^{1-s}|\Delta B|^{2} d x d \tau\right) \\
& \leq C\left(\mathcal{A}_{1}+\mathcal{A}_{3}\right)^{\frac{1}{2}} C_{0}^{\frac{1}{2}}\left(C_{0}+C_{0}^{\theta}\right) .
\end{aligned}
$$

Hence we obtain

$$
\mathcal{A}_{2} \leq C C_{0}^{\frac{1}{2}}\left(\mathcal{A}_{1}+\mathcal{A}_{3}\right)\left(C_{0}+C_{0}^{\theta}\right)+C C_{0}+C\left(\mathcal{A}_{1}+\mathcal{A}_{3}\right)^{\frac{1}{2}} C_{0}^{\frac{1}{2}}\left(C_{0}+C_{0}^{\theta}\right)
$$

It remains to estimate $\mathcal{A}_{3}$. For $s \in\left(\frac{1}{2}, 1\right]$, we define $r=\frac{6}{3-2 s}$, then we have $r \in(3,6]$. Simple computations yield

$$
\frac{6-r}{2 r}=1-s, \quad \frac{3 r-6}{2 r}=s .
$$


18

ANTHONY SEEN

We bound the term $\tau^{2-s} \int_{\mathbb{R}^{3}}|\nabla u|^{2}|B|^{2} d x$ as follows. Since $r>3$, we can apply (1.16) to obtain

$$
\begin{aligned}
& \tau^{2-s} \int_{\mathbb{R}^{3}}|\nabla u|^{2}|B|^{2} d x \\
& \leq \tau\left(\tau^{1-s} \int_{\mathbb{R}^{3}}|\nabla u|^{2} d x\right)\|B(\cdot, \tau)\|_{L^{\infty}}^{2} \\
& \leq C \tau\left(\tau^{1-s} \int_{\mathbb{R}^{3}}|\nabla u|^{2} d x\right)\left(\int_{\mathbb{R}^{3}}|B|^{r} d x+\int_{\mathbb{R}^{3}}|\nabla B|^{r} d x\right)^{\frac{2}{r}} .
\end{aligned}
$$

The term $\|B\|_{L^{r}}$ can be bounded by the $L^{2}-L^{6}$ interpolation on $B$. Hence using (1.14) and the bound (2.7), we obtain

$$
\begin{aligned}
& \tau^{2-s} \int_{\mathbb{R}^{3}}|\nabla u|^{2}|B|^{2} d x \\
& \leq C \tau C_{0}^{3}+C \tau\left(\tau^{1-s} \int_{\mathbb{R}^{3}}|\nabla u|^{2} d x\right)\left(\int_{\mathbb{R}^{3}}|\nabla B|^{2} d x\right)^{\frac{6-r}{2 r}}\left(\int_{\mathbb{R}^{3}}|\Delta B|^{2} d x\right)^{\frac{3 r-6}{2 r}} \\
& \leq C \tau C_{0}^{3}+C\left(\tau^{1-s} \int_{\mathbb{R}^{3}}|\nabla u|^{2} d x\right)\left(\tau^{1-s} \int_{\mathbb{R}^{3}}|\nabla B|^{2} d x\right)^{1-s}\left(\tau^{2-s} \int_{\mathbb{R}^{3}}|\Delta B|^{2} d x\right)^{s} \\
& \leq C \tau C_{0}^{3}+C C_{0} C_{0}^{1-s}\left(\tau^{2-s} \int_{\mathbb{R}^{3}}\left|B_{t}\right|^{2} d x+\int_{\mathbb{R}^{3}}\left(|\nabla B|^{2}|u|^{2}+|\nabla u|^{2}|B|^{2}\right) d x\right)^{s} \\
& \leq C \tau C_{0}^{3}+C C_{0} C_{0}^{1-s}\left(\mathcal{A}_{1}+\mathcal{A}_{3}\right)^{s} .
\end{aligned}
$$

By similar method, we obtain

$$
\begin{aligned}
\tau^{2-s} \int_{\mathbb{R}^{3}}|\nabla B|^{2}|B|^{2} d x & \leq t\left(\tau^{1-s} \int_{\mathbb{R}^{3}}|\nabla B|^{2} d x\right)\|B(\cdot, \tau)\|_{L^{\infty}}^{2} \\
& \leq C \tau C_{0}^{3}+C C_{0} C_{0}^{1-s}\left(\mathcal{A}_{1}+\mathcal{A}_{3}\right)^{s} .
\end{aligned}
$$

For the term $\tau^{2-s} \int_{\mathbb{R}^{3}}|\nabla B|^{2}|u|^{2} d x$, we have

$$
\begin{aligned}
\tau^{2-s} \int_{\mathbb{R}^{3}}|\nabla B|^{2}|u|^{2} d x & \leq \tau\left(\tau^{1-s} \int_{\mathbb{R}^{3}}|\nabla B|^{2} d x\right)\|u(\cdot, \tau)\|_{L^{\infty}}^{2} \\
& \leq C \tau\left(\tau^{1-s} \int_{\mathbb{R}^{3}}|\nabla B|^{2} d x\right)\left(\int_{\mathbb{R}^{3}}|u|^{r} d x+\int_{\mathbb{R}^{3}}|\nabla u|^{r} d x\right)^{\frac{2}{r}} .
\end{aligned}
$$

Similar to the case for $B$, the term $\|u\|_{L^{r}}$ can be bounded by the $L^{2}-L^{6}$ interpolation on $u$. For the term $\|\nabla u\|_{L^{r}}$, we can apply (2.8) with the bounds (2.6)-(2.7) to get

$$
\begin{aligned}
\tau\left(\int_{\mathbb{R}^{3}}|\nabla u|^{r} d x\right)^{\frac{2}{r}} & \leq C \tau\left(\int_{\mathbb{R}^{3}}\left(|F|^{r}+|\omega|^{r}+|\rho-\tilde{\rho}|^{r}\right) d x\right)^{\frac{2}{r}} \\
& \leq C\left(\tau^{1-s} \int_{\mathbb{R}^{3}}|F|^{2} d x\right)^{1-s}\left(\tau^{1-s} \int_{\mathbb{R}^{3}}|\nabla F|^{2} d x\right)^{s} \\
& +C\left(\tau^{1-s} \int_{\mathbb{R}^{3}}|\omega|^{2} d x\right)^{1-s}\left(\tau^{1-s} \int_{\mathbb{R}^{3}}|\nabla \omega|^{2} d x\right)^{s}+C\left(\int_{\mathbb{R}^{3}}|\rho-\tilde{\rho}|^{r} d x\right)^{\frac{2}{r}} \\
& \leq C C_{0}^{1-s}\left(\mathcal{A}_{1}+\mathcal{A}_{3}\right)^{s}+C\left(\int_{\mathbb{R}^{3}}|\rho-\tilde{\rho}|^{r} d x\right)^{\frac{2}{r}} .
\end{aligned}
$$


Together with the bound on $P-\tilde{P}$ given by (2.10), we obtain

$$
\begin{aligned}
& \tau^{2-s} \int_{\mathbb{R}^{3}}|\nabla B|^{2}|u|^{2} d x \\
& \leq C \tau C_{0}^{3}+C C_{0} C_{0}^{1-s}\left(\mathcal{A}_{1}+\mathcal{A}_{3}\right)^{s}+C C_{0} C_{0}^{\frac{2}{r}},
\end{aligned}
$$

and therefore by taking supremum over $\tau \in[0, T]$,

$$
\mathcal{A}_{3} \leq C C_{0}^{3}+C C_{0} C_{0}^{1-s}\left(\mathcal{A}_{1}+\mathcal{A}_{3}\right)^{s}+C C_{0} C_{0}^{\frac{2}{r}} .
$$

Combining the results (2.26), (2.27) and (2.28), and utilising the smallness condition on $C_{0}$, there is some $\theta>0$ such that 2.25) holds. In particular,

$$
\sup _{0 \leq \tau \leq T} \tau^{2-s} \int_{\mathbb{R}^{3}}\left(|\dot{u}|^{2}+\left|B_{t}\right|^{2}\right) d x+\int_{0}^{T} \int_{\mathbb{R}^{3}} \tau^{2-s}\left(|\nabla \dot{u}|^{2}+\left|\nabla B_{t}\right|^{2}\right) d x d \tau \leq C C_{0}^{\theta} .
$$

We finish the proof of (2.23).

By combining the estimates obtained in previous lemmas, we have the following estimates on $\mathcal{A}(T)$ when $T \leq 1$ under the pointwise bound (2.4) on $\rho$ and smallness assumption on $C_{0}$. In other words, for $C_{0} \ll 1$ and $T \leq 1$, we have

$$
\mathcal{A}(T) \leq C C_{0}^{\theta}
$$

for some $\theta>0$.

2.2. Estimates on $u$ and $B$ for $T>1$. In this subsection, we proceed to estimate $\mathcal{A}(T)$ when $T>1$. In view of the definition of $\mathcal{A}$ and the bounds (2.10) and (2.29), it suffices to bound the following term for $t>1$ :

$$
\begin{gathered}
\tilde{\mathcal{A}}(t)=\sup _{1 \leq \tau \leq t}\left[\int_{\mathbb{R}^{3}}\left(|\nabla u|^{2}+|\nabla B|^{2}+|\dot{u}|^{2}+\left|B_{t}\right|^{2}\right)\right](x, \tau) d x \\
+\int_{1}^{t} \int_{\mathbb{R}^{3}}\left(|\dot{u}|^{2}+\left|B_{t}\right|^{2}+|\nabla \dot{u}|^{2}+\left|\nabla B_{t}\right|^{2}\right) d x d \tau .
\end{gathered}
$$

Before we estimate $\tilde{\mathcal{A}}(t)$, we state the following estimates on $\rho$ which was proved in Hoff 10 .

Proposition 2.10. Assume that $\rho$ satisfies (2.4), then for $t>1$, we have

$$
\int_{1}^{t} \int_{\mathbb{R}^{3}}|\rho-\tilde{\rho}|^{4} d x d \tau \leq C \int_{1}^{t} \int_{\mathbb{R}^{3}}|F|^{4} d x d \tau+C C_{0}
$$

We further introduce the following auxiliary bounds (2.32)-(2.34) which will be used in controlling $\tilde{\mathcal{A}}(t)$. For simplicity, we define an auxiliary functional $\tilde{\mathcal{H}}(t)$ by

$$
\tilde{\mathcal{H}}(t)=\int_{1}^{t} \int_{\mathbb{R}^{3}}\left(|\nabla u|^{4}+|\nabla B|^{4}\right) d x d \tau .
$$

Lemma 2.11. Assume that $\rho$ satisfies (2.4), then for $t>1$, we have

$$
\begin{aligned}
& \int_{1}^{t} \int_{\mathbb{R}^{3}}\left(|u|^{4}+|B|^{4}\right) d x d \tau \leq C C_{0}^{\frac{3}{2}} \tilde{\mathcal{A}}(t)^{\frac{1}{2}}, \\
& \int_{1}^{t} \int_{\mathbb{R}^{3}}\left(|u|^{8}+|B|^{8}\right) d x d \tau \leq C C_{0}^{2} \tilde{\mathcal{A}}(t)^{2}+C C_{0}^{\frac{1}{2}} \tilde{\mathcal{A}}(t)^{\frac{3}{2}} \tilde{\mathcal{H}}(t), \\
& \sup _{1 \leq \tau \leq t}\left(\|u(\cdot, \tau)\|_{L^{\infty}}+\|B(\cdot, \tau)\|_{L^{\infty}}\right) \leq C\left(\tilde{\mathcal{A}}(t)^{2}+\tilde{\mathcal{A}}(t)^{\frac{1}{2}}+C_{0}^{\theta}\right),
\end{aligned}
$$


where $\theta>0$.

Proof. To prove (2.32), using (1.14), (2.10) and the definition of $\tilde{\mathcal{A}}(t)$, we can bound the term $\int_{1}^{t} \int_{\mathbb{R}^{3}}\left(|u|^{4}+|B|^{4}\right)$ as follows.

$$
\begin{aligned}
& \int_{1}^{t} \int_{\mathbb{R}^{3}}\left(|u|^{4}+|B|^{4}\right) d x d \tau \\
& \leq C \int_{1}^{T}\left(\int_{\mathbb{R}^{3}}|u|^{2} d x\right)^{\frac{1}{2}}\left(\int_{\mathbb{R}^{3}}|\nabla u|^{2} d x\right)^{\frac{3}{2}} d \tau+C \int_{1}^{T}\left(\int_{\mathbb{R}^{3}}|B|^{2} d x\right)^{\frac{1}{2}}\left(\int_{\mathbb{R}^{3}}|\nabla B|^{2} d x\right)^{\frac{3}{2}} d \tau \\
& \leq C C_{0}^{\frac{3}{2}} \tilde{\mathcal{A}}^{\frac{1}{2}} .
\end{aligned}
$$

Next to prove (2.33), using (1.16) and (2.32), we have

$$
\begin{aligned}
& \int_{1}^{t} \int_{\mathbb{R}^{3}}|u|^{8} d x d \tau \\
& \leq\left(\sup _{1 \leq \tau \leq t} \int_{\mathbb{R}^{3}}|u|^{4} d x\right) \int_{1}^{T}\|u(\cdot, \tau)\|_{L^{\infty}}^{4} d \tau \\
& \leq C\left(\sup _{1 \leq \tau \leq t} \int_{\mathbb{R}^{3}}|u|^{2} d x\right)^{\frac{1}{2}}\left(\sup _{1 \leq \tau \leq t} \int_{\mathbb{R}^{3}}|\nabla u|^{2} d x\right)^{\frac{3}{2}}\left(\int_{1}^{t} \int_{\mathbb{R}^{3}}\left(|u|^{4}+|\nabla u|^{4}\right) d x d \tau\right) \\
& \leq C C_{0}^{2} \tilde{\mathcal{A}}^{2}+C C_{0}^{\frac{1}{2}} \tilde{\mathcal{A}}^{\frac{3}{2}} \int_{1}^{t} \int_{\mathbb{R}^{3}}|\nabla u|^{4} d x d \tau,
\end{aligned}
$$

and similarly,

$$
\int_{1}^{t} \int_{\mathbb{R}^{3}}|B|^{8} d x d \tau \leq C C_{0}^{2} \tilde{\mathcal{A}}^{2}+C C_{0}^{\frac{1}{2}} \tilde{\mathcal{A}}^{\frac{3}{2}} \int_{1}^{t} \int_{\mathbb{R}^{3}}|\nabla B|^{4} d x d \tau .
$$

Finally, to show (2.34), we use (1.16), (2.8) and (2.10) to obtain, for $1 \leq \tau \leq t$,

$$
\begin{aligned}
& \|u(\cdot, \tau)\|_{L^{\infty}} \\
& \leq C\left(\int_{\mathbb{R}^{3}}|u|^{4} d x\right)^{\frac{1}{4}}+C\left(\int_{\mathbb{R}^{3}}|\nabla u|^{4} d x\right)^{\frac{1}{4}} \\
& \leq C\left(\int_{\mathbb{R}^{3}}|u|^{2} d x\right)^{\frac{1}{8}}\left(\int_{\mathbb{R}^{3}}|\nabla u|^{2} d x\right)^{\frac{3}{8}} \\
& \quad+C\left[\left(\int_{\mathbb{R}^{3}}|F|^{4} d x\right)^{\frac{1}{4}}+\left(\int_{\mathbb{R}^{3}}|\omega|^{4} d x\right)^{\frac{1}{4}}+\left(\int_{\mathbb{R}^{3}}|\rho-\tilde{\rho}|^{4} d x\right)^{\frac{1}{4}}\right] \\
& \quad \leq C C_{0}^{\frac{1}{8}} \tilde{\mathcal{A}}^{\frac{3}{8}}+C\left[\left(\int_{\mathbb{R}^{3}}|F|^{4} d x\right)^{\frac{1}{4}}+\left(\int_{\mathbb{R}^{3}}|\omega|^{4} d x\right)^{\frac{1}{4}}+C_{0}^{\frac{1}{4}}\right] .
\end{aligned}
$$

To estimate the term involving $F$, we apply (2.6) and (2.6) together with (1.14) to have

$$
\begin{aligned}
& \left(\int_{\mathbb{R}^{3}}|F|^{4} d x\right)^{\frac{1}{4}} \\
& \leq C\left(\int_{\mathbb{R}^{3}}|F|^{2} d x\right)^{\frac{1}{8}}\left(\int_{\mathbb{R}^{3}}|\nabla F|^{2} d x\right)^{\frac{3}{8}} \\
& \leq C\left(\int_{\mathbb{R}^{3}}\left(|\nabla u|^{2}+|\rho-\tilde{\rho}|^{2}\right) d x\right)^{\frac{1}{8}}\left(\int_{\mathbb{R}^{3}}\left(|\dot{u}|^{2}+|\nabla B|^{2}|B|^{2}\right) d x\right)^{\frac{3}{8}} \\
& \leq C\left(\tilde{\mathcal{A}}+C_{0}\right)^{\frac{1}{8}}\left(\tilde{\mathcal{A}}+\|B(\cdot, \tau)\|_{L^{\infty}}^{2} \tilde{\mathcal{A}}\right)^{\frac{3}{8}} .
\end{aligned}
$$


The term involving $\omega$ can be estimated in a similar way to $F$, so we obtain

$$
\|u(\cdot, \tau)\|_{L^{\infty}} \leq C\left(C_{0}^{\frac{1}{8}} \tilde{\mathcal{A}}^{\frac{3}{8}}+\tilde{\mathcal{A}}^{\frac{1}{2}}+\tilde{\mathcal{A}}^{\frac{1}{2}}\|B(\cdot, \tau)\|_{L^{\infty}}^{\frac{3}{4}}+C_{0}^{\frac{1}{8}} \tilde{\mathcal{A}}^{\frac{3}{8}}\|B(\cdot, \tau)\|_{L^{\infty}}^{\frac{3}{4}}+C_{0}^{\frac{1}{4}}\right) .
$$

For $\|B(\cdot, \tau)\|_{L^{\infty}}$, we can estimate it as follows.

$$
\begin{aligned}
& \|B(\cdot, \tau)\|_{L^{\infty}} \\
& \leq C\left(\int_{\mathbb{R}^{3}}|B|^{4} d x\right)^{\frac{1}{4}}+C\left(\int_{\mathbb{R}^{3}}|\nabla B|^{4} d x\right)^{\frac{1}{4}} \\
& \leq C\left(\int_{\mathbb{R}^{3}}|B|^{2} d x\right)^{\frac{1}{8}}\left(\int_{\mathbb{R}^{3}}|\nabla B|^{2} d x\right)^{\frac{3}{8}}+C\left(\int_{\mathbb{R}^{3}}|\nabla B|^{2} d x\right)^{\frac{1}{8}}\left(\int_{\mathbb{R}^{3}}|\Delta B|^{2} d x\right)^{\frac{3}{8}} \\
& \leq C C_{0}^{\frac{1}{8}} \tilde{\mathcal{A}}^{\frac{3}{8}}+C \tilde{\mathcal{A}}^{\frac{1}{8}}\left(\int_{\mathbb{R}^{3}}\left(\left|B_{t}\right|^{2}+|\nabla B|^{2}|u|^{2}+|\nabla u|^{2}|B|^{2}\right) d x\right)^{\frac{3}{8}} \\
& \leq C C_{0}^{\frac{1}{8}} \tilde{\mathcal{A}}^{\frac{3}{8}}+C \tilde{\mathcal{A}}^{\frac{1}{8}}\left(\tilde{\mathcal{A}}+\left(\|B\|_{L^{\infty}}+\|u\|_{L^{\infty}}\right)^{2} \tilde{\mathcal{A}}\right)^{\frac{3}{8}} \\
& \leq C C_{0}^{\frac{1}{8}} \tilde{\mathcal{A}}^{\frac{3}{8}}+C \tilde{\mathcal{A}}^{\frac{1}{2}}+C \tilde{\mathcal{A}}^{\frac{1}{2}}\left(\|B\|_{L^{\infty}}+\|u\|_{L^{\infty}}\right)^{\frac{3}{4}} .
\end{aligned}
$$

Therefore with the help of Cauchy's inequality, the bound (2.34) then follows.

We are now going to obtain the estimates on $\tilde{\mathcal{A}}(t)$. The main difference between here and the analysis given in subsection 2.1 is that, we cannot use the $L^{6}$ estimates on $u$ and $B$ in controlling $\tilde{\mathcal{A}}(t)$ for $t>1$. We therefore need to make use of some new methods which will be illustrated in Lemma 2.12 as below.

Lemma 2.12. Assume that $\rho$ satisfies (2.4). For $C_{0} \ll 1$ and $t>1$, we have

$$
\tilde{\mathcal{A}}(t) \leq C C_{0}^{\theta},
$$

for some $\theta>0$.

Proof. Following the similar argument given in the proof of Lemma 2.7, we have for $t \in[1, T]$ that

$$
\begin{aligned}
& \sup _{1 \leq \tau \leq t} \int_{\mathbb{R}^{3}}\left(|\nabla u|^{2}+|\nabla B|^{2}\right)(x, \tau) d x+\int_{1}^{t} \int_{\mathbb{R}^{3}}\left(|\dot{u}|^{2}+\left|B_{t}\right|^{2}\right) d x d \tau \\
& \leq \mathcal{A}(1)+\int_{1}^{t} \int_{\mathbb{R}^{3}}\left(|\nabla B|^{2}|B|^{2}+|\nabla u|^{2}|B|^{2}+|\nabla B|^{2}|u|^{2}\right) d x d \tau .
\end{aligned}
$$

Hence using (2.32), we obtain

$$
\begin{aligned}
& \int_{1}^{t} \int_{\mathbb{R}^{3}}\left(|\nabla B|^{2}|B|^{2}+|\nabla u|^{2}|B|^{2}+|\nabla B|^{2}|u|^{2}\right) d x d \tau \\
& \leq C \\
& \leq\left[\left(\int_{1}^{t} \int_{\mathbb{R}^{3}}|u|^{4} d x d \tau\right)^{\frac{1}{2}}+\left(\int_{1}^{t} \int_{\mathbb{R}^{3}}|B|^{4} d x d \tau\right)^{\frac{1}{2}}\right] \\
& \quad \times\left[\left(\int_{1}^{t} \int_{\mathbb{R}^{3}}|\nabla u|^{4} d x d \tau\right)^{\frac{1}{2}}+\left(\int_{1}^{t} \int_{\mathbb{R}^{3}}|\nabla B|^{4} d x d \tau\right)^{\frac{1}{2}}\right] \\
& \leq C C_{0}^{\frac{3}{4}} \tilde{\mathcal{A}}^{\frac{1}{4}} \tilde{\mathcal{H}}^{\frac{1}{2}},
\end{aligned}
$$


and thus

$$
\begin{aligned}
& \sup _{1 \leq \tau \leq t} \int_{\mathbb{R}^{3}}\left(|\nabla u|^{2}+|\nabla B|^{2}\right)(x, \tau) d x+\int_{1}^{t} \int_{\mathbb{R}^{3}}\left(|\dot{u}|^{2}+\left|B_{t}\right|^{2}\right) d x d \tau \\
& \leq \mathcal{A}(1)+C C_{0}^{\frac{3}{4}} \tilde{\mathcal{A}}^{\frac{1}{4}} \tilde{\mathcal{H}}^{\frac{1}{2}} .
\end{aligned}
$$

On the other hand, following the argument given in the proof of Lemma 2.9. we have, for $\in[1, T]$ that

$$
\begin{aligned}
& \sup _{1 \leq \tau \leq t} \int_{\mathbb{R}^{3}}\left(|\dot{u}|^{2}+\left|B_{t}\right|^{2}\right)(x, \tau) d x+\int_{1}^{t} \int_{\mathbb{R}^{3}}\left(|\nabla \dot{u}|^{2}+\left|\nabla B_{t}\right|^{2}\right) d x d \tau \\
& \leq \mathcal{A}(1)+C \int_{1}^{t} \int_{\mathbb{R}^{3}}|B|^{2}|u|^{2}\left(|\nabla u|^{2}+|\nabla B|^{2}\right) d x d \tau \\
& \quad+C \int_{1}^{t} \int_{\mathbb{R}^{3}}\left(|B|^{2}\left|B_{t}\right|^{2}+|u|^{2}|\dot{u}|^{2}+\left|B_{t}\right|^{2}|u|^{2}\right) d x d \tau \\
& \quad+C \int_{1}^{t} \int_{\mathbb{R}^{3}}\left(|\nabla u|^{4}+|\nabla B|^{4}\right) d x d \tau .
\end{aligned}
$$

The term $\int_{1}^{t} \int_{\mathbb{R}^{3}}|B|^{2}|u|^{2}\left(|\nabla u|^{2}+|\nabla B|^{2}\right) d x d \tau$ can be estimated as follows.

$$
\begin{aligned}
& \int_{1}^{t} \int_{\mathbb{R}^{3}}|B|^{2}|u|^{2}\left(|\nabla u|^{2}+|\nabla B|^{2}\right) d x d \tau \\
& \leq \int_{1}^{t} \int_{\mathbb{R}^{3}}\left(|u|^{8}+|B|^{8}\right) d x d \tau+\int_{1}^{t} \int_{\mathbb{R}^{3}}\left(|\nabla u|^{4}+|\nabla B|^{4}\right) d x d \tau .
\end{aligned}
$$

The term $\int_{1}^{t} \int_{\mathbb{R}^{3}}\left(|u|^{8}+|B|^{8}\right) d x d \tau$ can be bounded using (2.33), and to bound the term $\int_{1}^{t} \int_{\mathbb{R}^{3}}|B|^{2}\left|B_{t}\right|^{2} d x d \tau$, we can use (1.14) and (2.10) to get

$$
\begin{aligned}
& \int_{1}^{t} \int_{\mathbb{R}^{3}}|B|^{2}\left|B_{t}\right|^{2} d x d \tau \\
& \leq \int_{1}^{T}\left(\int_{\mathbb{R}^{3}}|B|^{2} d x\right)^{\frac{1}{4}}\left(\int_{\mathbb{R}^{3}}|\nabla B|^{2} d x\right)^{\frac{3}{4}}\left(\int_{\mathbb{R}^{3}}\left|B_{t}\right|^{2} d x\right)^{\frac{1}{4}}\left(\int_{\mathbb{R}^{3}}\left|\nabla B_{t}\right|^{2} d x\right)^{\frac{3}{4}} d \tau \\
& \leq C C_{0}^{\frac{1}{2}} \tilde{\mathcal{A}}^{\frac{3}{2}} .
\end{aligned}
$$

The terms $\int_{1}^{t} \int_{\mathbb{R}^{3}}\left|B_{t}\right|^{2}|u|^{2} d x d \tau$ and $\int_{1}^{t} \int_{\mathbb{R}^{3}}\left|B_{t}\right|^{2}|u|^{2} d x d \tau$ can be treated in a similar way which gives

$$
\int_{1}^{t} \int_{\mathbb{R}^{3}}\left(|B|^{2}\left|B_{t}\right|^{2}+|u|^{2}|\dot{u}|^{2}+\left|B_{t}\right|^{2}|u|^{2}\right) d x d \tau \leq C C_{0}^{\frac{1}{2}} \tilde{\mathcal{A}}^{\frac{3}{2}} .
$$

Therefore we have

$$
\begin{aligned}
& \sup _{1 \leq \tau \leq t} \int_{\mathbb{R}^{3}}\left(|\dot{u}|^{2}+\left|B_{t}\right|^{2}\right)(x, \tau) d x+\int_{1}^{t} \int_{\mathbb{R}^{3}}\left(|\nabla \dot{u}|^{2}+\left|\nabla B_{t}\right|^{2}\right) d x d \tau \\
& \leq \mathcal{A}(1)+C C_{0}^{2} \tilde{\mathcal{A}}^{2}+C C_{0}^{\frac{1}{2}} \tilde{\mathcal{A}}^{\frac{3}{2}} \tilde{\mathcal{H}}+C C_{0}^{\frac{1}{2}} \tilde{\mathcal{A}}^{\frac{3}{2}}+C \tilde{\mathcal{H}} .
\end{aligned}
$$

It remains to estimate $\tilde{\mathcal{H}}$. Using (1.16), (2.8), (2.10) and (2.31), we have

$$
\int_{1}^{t} \int_{\mathbb{R}^{3}}|\nabla u|^{4} d x d \tau \leq C\left(\int_{1}^{t} \int_{\mathbb{R}^{3}}|F|^{4} d x d \tau+\int_{1}^{t} \int_{\mathbb{R}^{3}}|\omega|^{4} d x d \tau\right)+C C_{0} .
$$


To control $\int_{1}^{t} \int_{\mathbb{R}^{3}}|F|^{4} d x d \tau$, we use (1.14) to get

$$
\begin{aligned}
& \int_{1}^{t} \int_{\mathbb{R}^{3}}|F|^{4} d x d \tau \\
& \leq C \int_{1}^{T}\left(\int_{\mathbb{R}^{3}}|F|^{2} d x\right)^{\frac{1}{2}}\left(\int_{\mathbb{R}^{3}}|\nabla F|^{2} d x\right)^{\frac{3}{2}} d \tau \\
& \leq C\left(\sup _{1 \leq \tau \leq t} \int|\nabla F|^{2} d x\right)^{\frac{1}{2}}\left(\sup _{1 \leq \tau \leq t} \int|F|^{2} d x\right)^{\frac{1}{2}}\left(\int_{1}^{t} \int_{\mathbb{R}^{3}}|\nabla F|^{2} d x d \tau\right)^{\frac{1}{2}} .
\end{aligned}
$$

The term $\left(\sup _{1 \leq \tau \leq t} \int|F|^{2} d x\right)^{\frac{1}{2}}$ can be bounded by $C\left(\tilde{\mathcal{A}}+C_{0}\right)^{\frac{1}{2}}$, and using (2.36), the term $\left(\int_{1}^{t} \int_{\mathbb{R}^{3}}|\nabla F|^{2} d x d \tau\right)^{\frac{1}{2}}$ can be bound by

$$
C\left(\int_{1}^{t} \int_{\mathbb{R}^{3}}|\dot{u}|^{2} d x d \tau+\int_{1}^{t} \int_{\mathbb{R}^{3}}|\nabla B|^{2}|B|^{2} d x d \tau\right)^{\frac{1}{2}} \leq C\left(\tilde{\mathcal{A}}^{\frac{1}{2}}+C_{0}^{\frac{3}{8}} \tilde{\mathcal{A}}^{\frac{1}{8}} \tilde{\mathcal{H}}^{\frac{1}{4}}\right) .
$$

For $\left(\sup _{1 \leq \tau \leq t} \int|\nabla F|^{2} d x\right)^{\frac{1}{2}}$, we use (2.34) to otbain

$$
\begin{aligned}
\left(\sup _{1 \leq \tau \leq t} \int|\nabla F|^{2} d x\right)^{\frac{1}{2}} & \leq C\left(\sup _{1 \leq \tau \leq t} \int|\dot{u}|^{2} d x+\sup _{1 \leq \tau \leq t} \int|\nabla B|^{2}|B|^{2} d x\right)^{\frac{1}{2}} \\
& \leq C\left(\tilde{\mathcal{A}}+\sup _{1 \leq \tau \leq t}\|B(\cdot, \tau)\|_{L^{\infty}}^{2} \int_{\mathbb{R}^{3}}|\nabla B|^{2} d x\right)^{\frac{1}{2}} \\
& \leq C\left(\tilde{\mathcal{A}}+\left(\tilde{\mathcal{A}}(t)^{2}+\tilde{\mathcal{A}}(t)^{\frac{1}{2}}+C_{0}^{\theta}\right)^{2} \tilde{\mathcal{A}}\right)^{\frac{1}{2}}
\end{aligned}
$$

The term $\int_{1}^{t} \int_{\mathbb{R}^{3}}|\omega|^{4} d x d \tau$ can be estimated in a similar way, and we have

$$
\begin{aligned}
& \int_{1}^{t} \int_{\mathbb{R}^{3}}|\nabla u|^{4} d x d \tau \\
& \leq C C_{0}+C\left(\tilde{\mathcal{A}}+C_{0}\right)^{\frac{1}{2}}\left(\tilde{\mathcal{A}}+\left(\tilde{\mathcal{A}}(t)^{2}+\tilde{\mathcal{A}}(t)^{\frac{1}{2}}+C_{0}^{\theta}\right)^{2} \tilde{\mathcal{A}}\right)^{\frac{1}{2}}\left(\tilde{\mathcal{A}}^{\frac{1}{2}}+C_{0}^{\frac{3}{8}} \tilde{\mathcal{A}}^{\frac{1}{8}} \tilde{\mathcal{H}}^{\frac{1}{4}}\right) .
\end{aligned}
$$

We estimate $\int_{1}^{t} \int_{\mathbb{R}^{3}}|\nabla u|^{4}$ in a similar way as the case for $\nabla u$ and get

$$
\begin{aligned}
& \int_{1}^{t} \int_{\mathbb{R}^{3}}|\nabla B|^{4} d x d \tau \\
& \leq C \int_{1}^{T}\left(\int_{\mathbb{R}^{3}}|\nabla B|^{2} d x\right)^{\frac{1}{2}}\left(\int_{\mathbb{R}^{3}}|\Delta B|^{2} d x\right)^{\frac{3}{2}} d \tau \\
& \leq C \int_{1}^{T}\left(\int_{\mathbb{R}^{3}}|\nabla B|^{2} d x\right)^{\frac{1}{2}}\left(\int_{\mathbb{R}^{3}}\left(\left|B_{t}\right|^{2}+|B|^{2}|\nabla u|^{2}+|\nabla B|^{2}|u|^{2}\right) d x\right)^{\frac{3}{2}} d \tau \\
& \leq C\left(\tilde{\mathcal{A}}+C_{0}\right)^{\frac{1}{2}}\left(\tilde{\mathcal{A}}+\left(\tilde{\mathcal{A}}(t)^{2}+\tilde{\mathcal{A}}(t)^{\frac{1}{2}}+C_{0}^{\theta}\right)^{2} \tilde{\mathcal{A}}\right)^{\frac{1}{2}}\left(\tilde{\mathcal{A}}^{\frac{1}{2}}+C_{0}^{\frac{3}{8}} \tilde{\mathcal{A}}^{\frac{1}{8}} \tilde{\mathcal{H}}^{\frac{1}{4}}\right) .
\end{aligned}
$$

Using Cauchy's inequality, there are constants $\bar{\theta}>1$ and $\theta>0$ such that

$$
\tilde{\mathcal{H}} \leq C\left(\tilde{\mathcal{A}}^{\bar{\theta}}+C_{0}^{\theta}\right)
$$


By combining (2.37), (2.38), 2.39), replacing $\bar{\theta}>1$ and $\theta>0$ if necessary, we conclude that, for all $t \in[1, T]$ that

$$
\tilde{\mathcal{A}}(t) \leq C\left(\tilde{\mathcal{A}}(t)^{\bar{\theta}}+C_{0}^{\theta}\right)+C \mathcal{A}(1) .
$$

Using the bound (2.29) on $\mathcal{A}(1)$ and making use of the smallness assumption on $C_{0}$, the result (2.35) then follows.

Combining (2.29) and (2.35), under the assumption (2.4) and $C_{0} \ll 1$, we have for all $T>0$ that

$$
\mathcal{A}(T) \leq C C_{0}^{\theta}
$$

2.3. Pointwise bound on $\rho$ and proof of Theorem [2.2, We now close the estimates on $\mathcal{A}$ by proving the following pointwise bounds on $\rho$. Together with (2.40), it will finish the proof of Theorem 2.2

Lemma 2.13. For $C_{0} \ll 1$, we have the pointwise bounds for $\rho$, namely

$$
\frac{1}{2} \rho_{1} \leq \rho(x, t) \leq 2 \rho_{2},
$$

for all $t \in[0, T]$ and $x \in \mathbb{R}^{3}$.

Proof. The proof is reminiscent of the one given in 22 which consists of a maximumprinciple argument applied along integral curves of the velocity field $u$, and we only give the sketch here. First we fix $y \in \mathbb{R}^{3}$ and define the corresponding integral curve of $u$ by

$$
\left\{\begin{array}{l}
\dot{x}(t)=u(x(t), t) \\
x(0)=y
\end{array}\right.
$$

From the definition (1.7) of $F$ and the mass equation (1.1) that

$$
(\mu+\lambda) \frac{d}{d t}[\log \rho(x(t), t)-\log (\tilde{\rho})]+P(\rho(x(t), t))-\tilde{P}=-F(x(t), t) .
$$

Integrating from $t_{0}$ to $t_{1}$ for $t_{1}, t_{2} \in[0, T]$, and abbreviating $\rho(x(t), t)$ by $\rho(t)$, etc., we then obtain

$$
\left.(\mu+\lambda)[\log \rho(s)-\log (\tilde{\rho})]\right|_{t_{0}} ^{t_{1}}+\int_{t_{0}}^{t_{1}}[P(\tau)-\tilde{P}] d \tau=-\int_{t_{0}}^{t_{1}} F(\tau) d \tau
$$

Since $P$ is increasing, the integral of $P$ on the left side of (2.43) is thus a dissipative term. On the other hand, if we can prove that

$$
\int_{t_{0}}^{t_{1}} F(\tau) d \tau \leq C C_{0}^{\theta^{\prime}}
$$

for some $\theta^{\prime}>0$, then by stipulating the smallness condition on $C_{0}$, we can see that the density $\rho$ should remain inside the interval $\left[\frac{1}{2} \rho_{1}, 2 \rho_{2}\right]$ for all $t \in[0, T]$, provided that the initial density satisfies $\rho_{0}(x) \in\left[\rho_{1}, \rho_{2}\right]$ for $x \in \mathbb{R}^{3}$. To see why (2.44) holds, 
using the Poisson equation (1.8), we can rewrite the integral of $F$ as

$$
\begin{aligned}
\int_{t_{0}}^{t_{1}} F(\tau) d \tau= & \int_{t_{0}}^{t_{1}}\left(\Gamma_{x_{j}} * g^{j}\right)(\cdot, \tau)(x(\tau)) d \tau \\
= & \int_{t_{0}}^{t_{1}} \int_{\mathbb{R}^{3}} \Gamma_{x_{j}}(x(\tau)-y) \rho \dot{u}^{j}(y, \tau) d y d \tau \\
& \quad-\int_{t_{0}}^{t_{1}} \int_{\mathbb{R}^{3}} \Gamma_{x_{j}}(x(\tau)-y)\left[\operatorname{div}\left(B B^{j}(y, \tau)\right)-B \cdot B_{x_{j}}(y, \tau)\right] d y d \tau .
\end{aligned}
$$

The integrals on the right here can be bounded using the estimates on the Hölder's norm of $u$ (which are given in Lemma 3.2) and a time integral on $\|B \cdot \nabla B(\cdot, t)\|_{L^{4}}$, and hence (2.44) holds. The argument can be made rigorous and we refer to 22$]$ for more details.

\section{Proof of Theorem 1.3}

In this section, we give the proof of Theorem 1.3 We fix the constants $d$ and $C$ defined in Theorem 2.2. We let initial data $\left(\rho_{0}, u_{0}, B_{0}\right)$ be given satisfying the hypotheses (1.17)-11.19) and take $\left(\rho_{0}^{\varepsilon}, u_{0}^{\varepsilon}, B_{0}^{\varepsilon}\right)$ to be smooth approximate initial data obtained by convolving $\left(\rho_{0}, u_{0}, B_{0}\right)$ with a standard mollifying kernel of width $\varepsilon>0$. Then by Theorem 2.1 as described in Section 2. for each $\varepsilon$, under the smallness assumption (1.19) on $C_{0}$ there is a corresponding smooth local solution $\left(\rho^{\varepsilon}, u^{\varepsilon}, B^{\varepsilon}\right)$ satisfying the bounds (2.3)-(2.4).

We recall the following theorem which shows that the smooth local solution $\left(\rho^{\varepsilon}, u^{\varepsilon}, B^{\varepsilon}\right)$ as described above exists on all of $\mathbb{R}^{3} \times[0, \infty)$. The proof can be found in 22] pp. $51-56$.

Theorem 3.1. Assume that the system parameters in (1.1)-(1.4) satisfy the conditions in (1.9)-(1.10), and let $d, \theta, C$ be as described in Theorem 1.3. Then given initial data $\left(\rho_{0}-\tilde{\rho}, u_{0}, B_{0}\right) \in H^{3}\left(\mathbb{R}^{3}\right)$ satisfying (1.17)-(1.19), the corresponding smooth solution $(\rho, u, B)$ described in Theorem 2.1 exists on all of $\mathbb{R}^{3} \times[0, \infty)$.

Using the estimates (2.3)-(2.4) from Theorem2.2 we obtain bounds on $\left(\rho^{\varepsilon}, u^{\varepsilon}, B^{\varepsilon}\right)$ which will provide the compactness needed to extract the desired global-in-time weak solution $(\rho, u, B)$ in the limit as $\varepsilon \rightarrow 0$. We first derive a result on the Höldercontinuity of $u^{\varepsilon}(\cdot, t), B^{\varepsilon}(\cdot, t), F^{\varepsilon}(\cdot, t)$ and $\omega^{\varepsilon}(\cdot, t)$ (here $F^{\varepsilon}$ and $\omega^{\varepsilon}$ are defined in terms of $\rho^{\varepsilon}, u^{\varepsilon}$ and $\left.B^{\varepsilon}\right)$.

Lemma 3.2. For $\alpha \in\left(0, \frac{1}{2}\right]$ and $t \in(0, \infty)$, we have the following estimates on the Hölder's norms on $u^{\varepsilon}, B^{\varepsilon}, F^{\varepsilon}$ and $\omega^{\varepsilon}$ : for any $\varepsilon>0$, we have

$$
\left\langle u^{\varepsilon}(\cdot, t)\right\rangle^{\alpha}+\left\langle B^{\varepsilon}(\cdot, t)\right\rangle^{\alpha}+\left\langle F^{\varepsilon}(\cdot, t)\right\rangle^{\alpha}+\left\langle\omega^{\varepsilon}(\cdot, t)\right\rangle^{\alpha} \leq C(t) C_{0}^{\theta},
$$

for some $\theta>0$ and $C(t)>0$ may depend on $t$ but independent of $\varepsilon$.

Proof. We only give the proof for $u^{\varepsilon}$ and the others are just similar. By the estimates (1.15) and (2.8),

$$
\left\langle u^{\varepsilon}(\cdot, t)\right\rangle^{\alpha} \leq C\left[\left\|F^{\varepsilon}(\cdot, t)\right\|_{L^{r}}+\left\|\omega^{\varepsilon}(\cdot, t)\right\|_{L^{r}}+\left\|\left(\rho^{\varepsilon}-\tilde{\rho}\right)(\cdot, t)\right\|_{L^{r}}\right] .
$$


On the other hand, by (1.14), we have

$$
\begin{aligned}
\left\|F^{\varepsilon}(\cdot, t)\right\|_{L^{r}} \leq C & \left(\left\|F^{\varepsilon}(\cdot, t)\right\|_{L^{2}}^{(6-r) / 2 r}\left\|\nabla F^{\varepsilon}(\cdot, t)\right\|_{L^{2}}^{(3 r-6) / 2 r}\right) \\
\leq C & \left(\left\|\left(\rho^{\varepsilon}-\tilde{\rho}\right)(\cdot, t)\right\|_{L^{2}}^{2}+\left\|\nabla u^{\varepsilon}(\cdot, t)\right\|_{L^{2}}^{2}\right)^{\frac{1-2 \alpha}{4}} \\
& \times\left(\left\|\dot{u}^{\varepsilon}(\cdot, t)\right\|_{L^{2}}^{2}+\left\|\nabla B^{\varepsilon} \cdot B^{\varepsilon}(\cdot, t)\right\|_{L^{2}}^{2}\right)^{\frac{1+2 \alpha}{4}} .
\end{aligned}
$$

The desired result then follows by applying the estimates (2.3)-(2.4).

Next we recall the following result on the Hölder-continuity of $u^{\varepsilon}(\cdot, t), B^{\varepsilon}(\cdot, t)$ in $x$ and $t$. It will be useful in obtaining uniform convergence of approximation solutions later. The proof can be found in 22 pp. 56 .

Proposition 3.3. Given $\tau>0$ there is a constant $C=C(\tau)$ independent of $\varepsilon$ such that,

$$
\left\langle u^{\varepsilon}\right\rangle_{\mathbb{R}^{3} \times[\tau, \infty)}^{\frac{1}{2}, \frac{1}{4}},\left\langle B^{\varepsilon}\right\rangle_{\mathbb{R}^{3} \times[\tau, \infty)}^{\frac{1}{2}, \frac{1}{4}} \leq C(\tau) C_{0}^{\theta} .
$$

The compactness of the approximate solutions $\left(\rho^{\varepsilon}, u^{\varepsilon}, B^{\varepsilon}\right)$ can then be summarised in the following lemma.

Lemma 3.4. There is a sequence $\varepsilon_{k} \rightarrow 0$ and functions $u, B$ and $\rho$ such that as $k \rightarrow \infty$,

$$
\begin{gathered}
u^{\varepsilon_{k}}, B^{\varepsilon_{k}} \rightarrow u, B \text { uniformly on compact sets in } \mathbb{R}^{3} \times(0, \infty) ; \\
\nabla u^{\varepsilon_{k}}(\cdot, t), \nabla B^{\varepsilon_{k}}(\cdot, t), \nabla \omega^{\varepsilon_{k}}(\cdot, t) \rightarrow \nabla u(\cdot, t), \nabla B(\cdot, t), \nabla \omega(\cdot, t)
\end{gathered}
$$

weakly in $L^{2}\left(\mathbb{R}^{3}\right)$ for all $t>0$;

$$
\sigma^{\frac{1-s}{2}} \dot{u}^{\varepsilon_{k}}, \sigma^{\frac{1-s}{2}} B_{t}^{\varepsilon_{k}}, \sigma^{\frac{2-s}{2}} \nabla \dot{u}^{\varepsilon_{k}}, \sigma^{\frac{2-s}{2}} \nabla B_{t}^{\varepsilon_{k}} \rightarrow \sigma^{\frac{1-s}{2}} \dot{u}, \sigma^{\frac{1-s}{2}} B_{t}, \sigma^{\frac{2-s}{2}} \nabla \dot{u}, \sigma^{\frac{2-s}{2}} \nabla B_{t}
$$

weakly in $L^{2}\left(\mathbb{R}^{3} \times[0, \infty)\right)$; and

$$
\rho^{\varepsilon_{k}}(\cdot, t) \rightarrow \rho(\cdot, t)
$$

strongly in $L_{\text {loc }}^{2}\left(\mathbb{R}^{3}\right)$ for every $t \geq 0$. Here $\sigma(t)=\min \{1, t\}$ and $s \in\left(\frac{1}{2}, 1\right]$.

Proof. The uniform convergence (3.3) follows from the bound (3.2) on $u$ and $B$ via a diagonal process, thus fixing the sequence $\left\{\varepsilon_{k}\right\}$. The statements in (3.4) and (3.5) then follow for this same sequence from (3.2) and considerations based on the equality of weak- $L^{2}$ derivatives and distribution derivatives. The strong convergence (3.6) of $\rho^{\varepsilon_{k}}$ for a further subsequence requires an argument given in Lions [16] which was later extended by Feireisl [8. We omit the details here.

Proof of Theorem 1.3. In view of Theorem 2.2. Proposition 3.3 and Lemma 3.4 the limiting functions $(\rho, u, B)$ of Lemma 3.4 inherit the bounds in (1.22)-(1.26). It is also clear from the modes of convergence described in Lemma 3.4 that $(\rho, u, B)$ satisfies the weak forms (1.11)-(1.13) of (1.1)-(1.4) as well as the initial condition (1.5). The continuity statement (1.20)-(1.21) then follows easily from these weak forms together with the bounds in (1.25)-(1.26).

It remains to prove the bound (1.28) on the piecewise $C^{\beta(t)}$ modulus of $\rho(\cdot)$ and time integral of $\|\nabla u(\cdot, t)\|_{L^{\infty}}$. We fix $T>0$ and $N>0$ such that

$$
\left\|\rho_{0}^{\varepsilon}-\tilde{\rho}\right\|_{C_{p w}^{\beta}} \leq N \text {. }
$$


We first decompose $u^{\varepsilon}$ as $u^{\varepsilon}=u_{F}^{\varepsilon}+u_{P}^{\varepsilon}$, where $u_{F}^{\varepsilon}$, $u_{P}^{\varepsilon}$ satisfy

$$
\left\{\begin{array}{l}
(\mu+\lambda) \Delta\left(u_{F}^{\varepsilon}\right)^{j}=F_{x_{j}}^{\varepsilon}+(\mu+\lambda)\left(\omega^{\varepsilon}\right)_{x_{k}}^{j, k} \\
(\mu+\lambda) \Delta\left(u_{P}^{\varepsilon}\right)^{j}=\left(P^{\varepsilon}-P(\tilde{\rho})\right)_{x_{j}} .
\end{array}\right.
$$

To bound $u_{F}^{\varepsilon}$ we apply the estimate (1.16) on $F^{\varepsilon}$ to obtain that, for any $r>3$ and $t \in[0, T]$,

$$
\int_{0}^{t}\left\|\nabla u_{F}^{\varepsilon}(\cdot, \tau)\right\|_{\infty} d \tau \leq C(r) \int_{0}^{t}\left[\left\|\nabla u_{F}^{\varepsilon}(\cdot, \tau)\right\|_{L^{r}}+\left\|D_{x}^{2} u_{F}^{\varepsilon}(\cdot, \tau)\right\|_{L^{r}}\right] d \tau .
$$

The right side of the above can be controlled by the time integrals on $\left\|\rho^{\varepsilon} \dot{u}^{\varepsilon}(\cdot, t)\right\|_{L^{r}}$ and $\left\|B^{\varepsilon} \cdot \nabla B^{\varepsilon}(\cdot, t)\right\|_{L^{r}}$, which can be bounded by the bounds (2.12) and (2.23). Therefore we obtain

$$
\int_{0}^{t}\left\|\nabla u_{F}^{\varepsilon}(\cdot, \tau)\right\|_{\infty} d \tau \leq C(t) C_{0}^{\theta}
$$

On the other hand, the way in controlling $u_{P}^{\varepsilon}$ is a bit more subtle than that of $u_{F}^{\varepsilon}$. For $t \in[0, T]$, the logical flow in obtaining the desired bound on $\int_{0}^{t}\left\|\nabla u_{P}^{\varepsilon}(\cdot, \tau)\right\|_{\infty} d \tau$ can be outlined as follows:

Step 1. $P^{\varepsilon}(\cdot, t)-\tilde{P}$ is pointwisely bounded independently of time.

Step 2. $u_{P}^{\varepsilon}(\cdot, t)$ is $\log$-Lipschitz with bounded log-Lipschitz seminorm.

Step 3. The integral curve $x^{\varepsilon}(y, t)$ as defined by

$$
\left\{\begin{array}{l}
\dot{x}^{\varepsilon}(t, y)=u^{\varepsilon}\left(x^{\varepsilon}(t, y), t\right) \\
x^{\varepsilon}(0, y)=y
\end{array}\right.
$$

is Hölder-continuous in $y$.

Step 4. $\rho^{\varepsilon}(\cdot, t)$ is piecewise $C^{\beta(t)}$ with exponent $\beta(t)$ and modulus suitably bounded in finite time.

Step 5. The $C^{\beta(t)+1}\left(\mathbb{R}^{3}\right)$ norm of $u_{P}^{\varepsilon}(\cdot, t)$ is finite in finite time.

Step 1 can be easily accomplished by making use of the pointwise bound (1.17) on the density. Then by using Step 1 and the Poisson equation (3.7) 2 on $u_{P}^{\varepsilon}$, we can apply the results from Bahouri-Chemin [2] to show that Step 2 holds for $u_{P}^{\varepsilon}$ with

$$
\left|u_{P}^{\varepsilon}(y, t)-u_{P}^{\varepsilon}(z, t)\right| \leq C m(|y-z|),
$$

where $C$ is a constant which depends only on $\bar{\rho}$ and $\tilde{\rho}$, and $m$ is given by

$$
m(z)=\left\{\begin{array}{lr}
z(1-\log (z)), & 0<z \leq 1 ; \\
z, & 1<z<\infty .
\end{array}\right.
$$

For Step 3, we apply (3.9) to obtain:

$$
\begin{aligned}
& \left|\frac{d}{d t}\right| x^{\varepsilon}(t, y)-\left.x^{\varepsilon}(t, z)\right|^{2} \mid \\
& \leq\left|x^{\varepsilon}(t, y)-x^{\varepsilon}(t, z)\right|\left|u_{P}^{\varepsilon}\left(x^{\varepsilon}(t, y), t\right)-u_{P}^{\varepsilon}\left(x^{\varepsilon}(t, z), t\right)\right| \\
& \quad \quad+\left|x^{\varepsilon}(t, y)-x^{\varepsilon}(t, z)\right|\left|u_{F}^{\varepsilon}\left(x^{\varepsilon}(t, y), t\right)-u_{F}^{\varepsilon}\left(x^{\varepsilon}(t, z), t\right)\right| \\
& \leq\left[C+|| \nabla u_{F}^{\varepsilon}(\cdot, t) \|_{\infty}\right]\left|x^{\varepsilon}(t, y)-x^{\varepsilon}(t, z)\right|^{2}\left[1-\log \left|x^{\varepsilon}(t, y)-x^{\varepsilon}(t, z)\right|^{2}\right] .
\end{aligned}
$$

Upon integrating the above differential inequality in time and utilizing the bound (3.8) on $u_{F}$, there exists $\beta_{1}(t), \beta_{2}(t), \tilde{C}(t)>0$ such that

$$
\left|x^{\varepsilon}(t, y)-x^{\varepsilon}(t, z)\right| \leq \tilde{C}(t)|y-z|^{\beta_{1}(t)}
$$

and

$$
|y-z| \leq \tilde{C}(t)\left|x^{\varepsilon}(t, y)-x^{\varepsilon}(t, z)\right|^{\beta_{2}(t)} .
$$


Next we proceed to Step 4. Let $y, z \in \mathbb{R}^{3}$ which are both on the same side of $\mathcal{C}(t)$. Then there exists $y_{0}, z_{0} \in \mathbb{R}^{3}$ such that

$$
\left\{\begin{array}{l}
x^{\varepsilon}\left(t, y_{0}\right)=y \\
x^{\varepsilon}\left(t, z_{0}\right)=z
\end{array}\right.
$$

and $y_{0}, z_{0}$ are both on the same side of $\mathcal{C}(0)$. Integrating the mass equation along integral curves $x^{\varepsilon}\left(t, y_{0}\right)$ and $x^{\varepsilon}\left(t, z_{0}\right)$, subtracting and recalling the definition (1.7) of $F$, we obtain that

$$
\begin{aligned}
\left|\log \rho^{\varepsilon}(y, t)-\log \rho^{\varepsilon}(z, t)\right| & \\
\leq\left|\log \rho_{0}^{\varepsilon}\left(y_{0}\right)-\log \rho_{0}^{\varepsilon}\left(z_{0}\right)\right|+ & \int_{0}^{t} \mid P\left(\rho_{0}^{\varepsilon}\left(x^{\varepsilon}\left(\tau, y_{0}\right), \tau\right)\right)-P\left(\rho^{\varepsilon}\left(x^{\varepsilon}\left(\tau, z_{0}\right), \tau\right) \mid d \tau\right. \\
& +\int_{0}^{t}\left|F^{\varepsilon}\left(x^{\varepsilon}\left(\tau, y_{0}\right), \tau\right)-F^{\varepsilon}\left(x^{\varepsilon}\left(\tau, z_{0}\right), \tau\right)\right| d \tau
\end{aligned}
$$

The first term on the right can be bounded in terms of $N$, and since $P$ is increasing, the second term is dissipative and can be dropped out. With the help of the estimate (2.7) on $F$ and the bound (3.10), the third term on the right is bounded as follows:

$$
\begin{aligned}
& \int_{0}^{t}\left|F^{\varepsilon}\left(x^{\varepsilon}\left(\tau, y_{0}\right), \tau\right)-F^{\varepsilon}\left(x^{\varepsilon}\left(\tau, z_{0}\right), \tau\right)\right| d \tau \\
& \quad \leq \int_{0}^{t}\left\|\nabla F^{\varepsilon}(\cdot, \tau)\right\|_{L^{r}}\left(\tilde{C}(t)\left|y_{0}-z_{0}\right|^{\beta_{1}(t)}\right)^{\alpha} d \tau \\
& \quad \leq C \int_{0}^{t}\left(\left\|\rho^{\varepsilon} \dot{u}^{\varepsilon}\right\|_{L^{r}}+\left\|B^{\varepsilon} \cdot \nabla B^{\varepsilon}\right\|_{L^{r}}\right)\left(\tilde{C}(t)\left|y_{0}-z_{0}\right|^{\beta_{1}(t)}\right)^{\alpha} d \tau,
\end{aligned}
$$

where $r>3$ and $\alpha=1-\frac{3}{r}$. Using the bounds in (2.3)-(2.4), the term involving $\dot{u}^{\varepsilon}$ can be bounded by

$$
\begin{aligned}
& C \int_{0}^{t}\left(\left\|\dot{u}^{\varepsilon}(\cdot, \tau)\right\|_{L^{2}}^{\frac{1-\delta}{2}}\left\|\nabla \dot{u}^{\varepsilon}(\cdot, \tau)\right\|_{L^{2}}^{\frac{1-\delta}{2}}\right) d \tau \\
& \leq C \int_{0}^{t} \tau^{\gamma}\left(\tau^{1-s} \int_{\mathbb{R}^{3}}\left|\dot{u}^{\varepsilon}\right|^{2} d x\right)^{\frac{1-\delta}{4}}\left(\tau^{2-s} \int_{\mathbb{R}^{3}}\left|\nabla \dot{u}^{\varepsilon}\right|^{2} d x\right)^{\frac{1+\delta}{4}} d \tau \\
& \leq C\left(\int_{0}^{t} \tau^{2 \gamma} d \tau\right)^{\frac{1}{2}} C^{\bar{\theta}},
\end{aligned}
$$

for some $\delta>0$ and $4 \gamma=(s-1)(1-\delta)-(2-s)$. Since $2 \gamma>-1$ if $s>\frac{1}{2}$, the above time integral is finite and hence we obtain

$$
\int_{0}^{t}\left\|\rho^{\varepsilon} \dot{u}^{\varepsilon}\right\|_{L^{r}}\left(\tilde{C}(t)\left|y_{0}-z_{0}\right|^{\beta_{1}(t)}\right)^{\alpha} d \tau \leq C(t)\left|y_{0}-z_{0}\right|^{\alpha \beta_{1}(t)},
$$

for some $C(t)>0$. The estimate for the term involving $B^{\varepsilon} \cdot \nabla B^{\varepsilon}$ is just similar, so by applying the bound (3.11), we obtain from (3.12) that,

$$
\begin{aligned}
\left|\log \rho^{\varepsilon}(y, t)-\log \rho^{\varepsilon}(z, t)\right| & \leq \tilde{C}(t) N|y-z|^{\beta_{0} \beta_{2}(t)}+\tilde{C}(t) C(t)|y-z|^{\alpha \beta_{1}(t) \beta_{2}(t)} \\
& \leq C\left(N, t, C_{0}\right)|y-z|^{\beta(t)}
\end{aligned}
$$

for some $C\left(N, t, C_{0}\right)>0$ and $\beta(t)>0$. The above shows that $\rho^{\varepsilon}(\cdot, t)$ is piecewise $C^{\beta(t)}$ with bounded modulus and Step 4 is completed.

Finally, with the improved regularity on $\rho^{\varepsilon}(\cdot, t)$ from Step 4 , we can now make use of $(3.7)_{2}$ again and apply properties of Newtonian potentials to conclude that 
the $C^{\beta(t)+1}\left(\mathbb{R}^{3}\right)$ norm of $u_{P}$ remains finite in finite time, which finishes Step 5 as described above.

We therefore obtain the bound on $u_{p}$ :

$$
\int_{0}^{t}\left\|\nabla u_{P}^{\varepsilon}(\cdot, \tau)\right\|_{\infty} d \tau \leq C\left(N, t, C_{0}\right) .
$$

for some $C\left(t, N, C_{0}\right)>0$. Combining (3.8), (3.13) and (3.14), there exists a constant $C\left(N, T, C_{0}\right)>0$ such that

$$
\sup _{0 \leq \tau \leq T}\left\|\rho^{\varepsilon}(\cdot, t)-\tilde{\rho}\right\|_{C_{p w}^{\beta(t)}}+\int_{0}^{T}\left\|\nabla u^{\varepsilon}(\cdot, \tau)\right\|_{L^{\infty}} d \tau \leq C\left(N, T, C_{0}\right)
$$

for all $\varepsilon>0$. Notice that $\beta$ and $C\left(N, T, C_{0}\right)$ are all independent of $\varepsilon$. Hence by taking $\varepsilon \rightarrow 0$ (or some subsequence $\varepsilon_{k} \rightarrow 0$ ), the limit $(\rho, u, B)$ satisfies (1.28) and we finish the proof of Theorem 1.3 .

\section{Uniqueness of Weak SOlution AND PRoof of Theorem 1.5}

In this section, we address the uniqueness of weak solutions to (1.1)-(1.4) and prove Theorem 1.5. To begin with, we fix constants $L, \beta_{0}, N, \rho_{1}, \rho_{2}, \tilde{\rho}>0, q>6$, $s \in\left(\frac{1}{2}, 1\right]$ and let $\left(\rho_{0}, u_{0}, B_{0}\right)$ and $\left(\bar{\rho}_{0}, \bar{u}_{0}, \bar{B}_{0}\right)$ be initial data satisfying (1.17)-(1.19) and (1.27). By Theorem 1.3. there exists weak solutions $(\rho, u, B)$ and $(\bar{\rho}, \bar{u}, \bar{B})$ to (1.1)-(1.4) with initial data $\left(\rho_{0}, u_{0}, B_{0}\right)$ and $\left(\bar{\rho}_{0}, \bar{u}_{0}, \bar{B}_{0}\right)$ respectively, both satisfying (1.20)-(1.26). In particular, for each $T>0$, we have

$$
\begin{gathered}
\overline{\mathcal{A}}(T)+\mathcal{A}(T) \leq C, \\
C^{-1} \leq\|\rho\|_{L^{\infty}\left(\mathbb{R}^{3} \times[0, T]\right)} \leq C, \quad C^{-1} \leq\|\bar{\rho}\|_{L^{\infty}\left(\mathbb{R}^{3} \times[0, T]\right)} \leq C, \\
\int_{0}^{T}\left(\|\nabla u(\cdot, \tau)\|_{L^{\infty}}+\|\nabla \bar{u}(\cdot, \tau)\|_{L^{\infty}}\right) d \tau \leq C,
\end{gathered}
$$

for some constant $C$ which may depend on $T, L, \beta_{0}, N, \rho_{1}, \rho_{2}, \tilde{\rho}, s$ and $C_{0}$, and $\overline{\mathcal{A}}(T)$ is defined by (2.5) with $(\rho, u, B)$ being replaced by $(\bar{\rho}, \bar{u}, \bar{B})$. We will then obtain the uniqueness and continuous dependence on initial data of weak solutions by proving the assertion (1.30) for $(\rho, u, B)$ and $(\bar{\rho}, \bar{u}, \bar{B})$.

We follow the idea and analysis given in Hoff 12 for compressible Navier-Stokes equations, which suggested that solutions with minimal regularity are best compared in a Lagrangian framework. We therefore state the following proposition about integral curves given in (2.42). More precisely, for $T>0$, the bound (4.3) guarantees the existence and uniqueness of the mapping $X\left(y, t, t^{\prime}\right) \in C\left(\mathbb{R}^{3} \times[0, T]^{2}\right)$ satisfying

$$
\left\{\begin{array}{l}
\frac{\partial X}{\partial t}\left(y, t, t^{\prime}\right)=u\left(X\left(y, t, t^{\prime}\right), t\right) \\
X\left(y, t^{\prime}, t^{\prime}\right)=y
\end{array}\right.
$$

where $(\rho, u, B)$ is a weak solution to (1.1)-(1.4) on $\mathbb{R}^{3} \times[0, T]$ satisfying (1.20)-(1.26) and (1.28). Moreover, using the bound (4.3), the mapping $X\left(\cdot, t, t^{\prime}\right)$ is Lipschitz on $\mathbb{R}^{3}$ for $\left(t, t^{\prime}\right) \in[0, T]^{2}$. 
Proposition 4.1. Let $T>0$ and $u$ satisfy 4.31). Then there is a unique function $X \in C\left(\mathbb{R}^{3} \times[0, T]^{2}\right)$ satisfying (4.4). In particular, $X\left(\cdot, t, t^{\prime}\right)$ is Lipschitz on $\mathbb{R}^{3}$ for $\left(t, t^{\prime}\right) \in[0, T]^{2}$, and there is a constant $C$ such that

$$
\left\|\frac{\partial X}{\partial y}\left(\cdot, t, t^{\prime}\right)\right\|_{L^{\infty}} \leq C, \quad\left(t, t^{\prime}\right) \in[0, T]^{2} .
$$

Proof. Refer to the proof of Lemma 2.1 in Hoff [12.

With respect to velocities $u$ and $\bar{u}$, for $y \in \mathbb{R}^{3}$, we let $X, \bar{X}$ be two integral curves given by

$$
\left\{\begin{array}{l}
\frac{\partial X}{\partial t}\left(y, t, t^{\prime}\right)=u\left(X\left(y, t, t^{\prime}\right), t\right) \\
X\left(y, t^{\prime}, t^{\prime}\right)=y
\end{array}\right.
$$

and

$$
\left\{\begin{array}{l}
\frac{\partial \bar{X}}{\partial t}\left(y, t, t^{\prime}\right)=\bar{u}\left(\bar{X}\left(y, t, t^{\prime}\right), t\right) \\
\bar{X}\left(y, t^{\prime}, t^{\prime}\right)=y
\end{array}\right.
$$

We then define $S(x, t), S^{-1}(x, t)$ by

$$
S(x, t)=\bar{X}(X(x, 0, t), t, 0)
$$

and

$$
S^{-1}(x, t)=X(\bar{X}(x, 0, t), t, 0) .
$$

The following proposition provides some properties of $S$ and $S^{-1}$ which will become useful later. A proof can be found in Hoff [12.

Proposition 4.2. Let $S$ and $S^{-1}$ be as given in (4.5)-(4.6). Then we have

- $S^{ \pm 1}$ is continuous on $R^{3} \times[0, T]$ and Lipschitz continuous on $R^{3} \times[\tau, T]$ for all $\tau>0$, and there is a constant $C$ such that

$$
\left\|\nabla S^{ \pm 1}(\cdot, t)\right\|_{L^{\infty}} \leq C, \quad t \in[0, T] ;
$$

- $\left(S_{t}+\nabla S u\right)(x, t)=\bar{u}(S(x, t), t)$ a.e. in $\mathbb{R}^{3} \times(0, T)$;

- $\bar{\rho}(S(x, t), t) \rho_{0}(X(x, 0, t)) \operatorname{det} \nabla S(x, t)=\rho(x, t) \bar{\rho}_{0}(X(x, 0, t))$ a.e. in $\mathbb{R}^{3} \times$ $(0, T)$.

We are now ready to give the proof of Theorem 1.5 First, we let $\psi: \mathbb{R}^{3} \times[0, T] \rightarrow$ $\mathbb{R}^{3}$ be a test function satisfying

$$
\begin{aligned}
& -\int_{\mathbb{R}^{3}} \rho_{0}(x) u_{0}(x) \psi(x, 0) d x \\
& =\int_{0}^{T} \int_{\mathbb{R}^{3}}\left[\rho u \cdot\left(\psi_{t}+\nabla \psi u\right)+(P(\rho)-\tilde{P}) \operatorname{div}(\psi)-\mu \nabla u^{j} \cdot \nabla \psi^{j}\right. \\
& \left.\quad-\lambda \operatorname{div}(u) \operatorname{div}(\psi)+\left(\frac{1}{2}|B|^{2}\right) \operatorname{div}(\psi)-B^{j} B \cdot \nabla \psi^{j}\right] d x d \tau .
\end{aligned}
$$


Define $\bar{\psi}=\psi \circ S^{-1}$. Then we have

$$
\begin{aligned}
- & \int_{\mathbb{R}^{3}} \bar{\rho}_{0}(x) \bar{u}_{0}(x) \bar{\psi}(x, 0) d x \\
=\int_{0}^{T} \int_{\mathbb{R}^{3}}\left[\overline { \rho } \overline { u } \cdot \left(\bar{\psi}_{t}\right.\right. & +\nabla \bar{\psi} \bar{u})+(P(\bar{\rho})-\tilde{P}) \operatorname{div}(\bar{\psi})-\mu \nabla \bar{u}^{j} \cdot \nabla \bar{\psi}^{j} \\
& \left.-\lambda \operatorname{div}(\bar{u}) \operatorname{div}(\bar{\psi})+\left(\frac{1}{2}|\bar{B}|^{2}\right) \operatorname{div}(\bar{\psi})-\bar{B}^{j} \bar{B} \cdot \nabla \bar{\psi}^{j}\right] d x d \tau
\end{aligned}
$$

Notice that

$$
\begin{aligned}
\int_{\mathbb{R}^{3}} \bar{\rho} \bar{u} \cdot\left(\bar{\psi}_{t}+\nabla \bar{\psi} \bar{u}\right) d x & =\int_{\mathbb{R}^{3}} \bar{\rho}(S) \bar{u}(S) \cdot\left(\bar{\psi}_{t}(S)+\nabla \bar{\psi} \bar{u}(S)\right)|\operatorname{det}(\nabla S)| d x \\
& =\int_{\mathbb{R}^{3}} A_{0} \rho \bar{u}(S)\left(\psi_{t}+\nabla \psi u\right) d x
\end{aligned}
$$

where we used the fact that $A_{0} \rho=(\bar{\rho} \circ S)|\operatorname{det}(\nabla S)|$ from Proposition 4.2. Hence by taking the difference between (4.7) and (4.8), and using the effective viscous flux $\bar{F}$ as defined in (1.7) (replacing $F$ by $\bar{F}, u$ by $\bar{u}$, etc.), for all $\psi$ and $\bar{\psi}$, we have

$$
\begin{aligned}
& \int_{\mathbb{R}^{3}}\left(\bar{\rho} \bar{u}_{0}-\rho_{0} u_{0}\right) \cdot \psi(x, 0) d x \\
& =\int_{0}^{T} \int_{\mathbb{R}^{3}}\left[\rho(u-\bar{u} \circ S)\left(\psi_{t}+\nabla \psi u\right)+\left(1-A_{0}\right) \rho(\bar{u} \circ S)\left(\psi_{t}+\nabla \psi u\right)\right] d x d \tau \\
& \quad+\int_{0}^{T} \int_{\mathbb{R}^{3}}\left[(\tilde{P}-P(\rho)) \operatorname{div}(\bar{\psi})+\mu \nabla \bar{u}^{j} \cdot \nabla \bar{\psi}^{j}+\lambda \operatorname{div}(\bar{u}) \operatorname{div}(\bar{\psi})\right] d x d \tau \\
& \quad+\int_{0}^{T} \int_{\mathbb{R}^{3}}\left[\left(1-A_{0}\right) \rho(\bar{u} \circ S)\left(\psi_{t}+\nabla \psi u\right)+(P(\rho)-P(\bar{\rho})) \operatorname{div}(\psi)\right] d x d \tau \\
& \quad+\int_{0}^{T} \int_{\mathbb{R}^{3}}\left(\frac{1}{2}|B|^{2} \operatorname{div}(\psi)-\frac{1}{2}|\bar{B}|^{2} \operatorname{div}(\bar{\psi})\right)-\left(B^{j} B \cdot \nabla \psi^{j}-\bar{B}^{j} \bar{B} \cdot \nabla \bar{\psi}^{j}\right) d x d \tau \\
& \quad+\int_{0}^{T} \int_{\mathbb{R}^{3}}\left[\nabla \bar{F} \cdot\left(\psi-\psi \circ S^{-1}\right)+\mu \bar{\omega}_{x_{k}}^{j, k}\left(\psi^{j}-\psi^{j} \circ S^{-1}\right)\right] d x d \tau \\
& \quad+\int_{0}^{T} \int_{\mathbb{R}^{3}}(\bar{u} \circ S-\bar{u})(\mu \Delta \psi+\lambda \nabla \operatorname{div}(\psi)) d x d \tau .
\end{aligned}
$$

Similarly, with respect to the magnetic fields $B$ and $\bar{B}$, we let $\varphi: \mathbb{R}^{3} \times[0, T] \rightarrow \mathbb{R}^{3}$ be test function satisfying

$$
\begin{aligned}
& -\int_{\mathbb{R}^{3}}\left(\bar{B}_{0}-B_{0}(x)\right) \cdot \varphi(x, 0) d x \\
& =\int_{0}^{T} \int_{\mathbb{R}^{3}}(B-\bar{B}) \cdot\left(\varphi_{t}+u \cdot \nabla \varphi+\nu \Delta \varphi\right) d x d \tau+\int_{0}^{T} \int_{\mathbb{R}^{3}} \nabla \varphi^{j}(\bar{B}-B) \bar{B}^{j} d x d \tau \\
& \quad+\int_{0}^{T} \int_{\mathbb{R}^{3}} \nabla \varphi^{j}\left(\bar{u}^{j}-u^{j}\right) B d x d \tau+\int_{0}^{T} \int_{\mathbb{R}^{3}} \nabla \varphi^{j}(u-\bar{u}) \bar{B}^{j} d x d \tau .
\end{aligned}
$$

Next we extend $\rho, u$ and $B$ to be constant in $t$ outside $[0, T]$ and let $\rho^{\varepsilon}, u^{\varepsilon}$ and $B^{\varepsilon}$ be the corresponding smooth approximation obtained by mollifying in both $x$ and $t$. Then we define $\psi^{\varepsilon}, \varphi^{\varepsilon}: \mathbb{R}^{3} \times[0, T] \rightarrow \mathbb{R}^{3}$ to be the solutions satisfying

$$
\left\{\begin{array}{l}
\rho^{\varepsilon}\left(\psi_{t}^{\varepsilon}+u^{\varepsilon} \cdot \nabla \psi^{\varepsilon}\right)+\mu \Delta \psi^{\varepsilon}+\lambda \nabla \operatorname{div}\left(\psi^{\varepsilon}\right)=G \\
\psi^{\varepsilon}(\cdot, T)=0
\end{array}\right.
$$


and

$$
\left\{\begin{array}{l}
\varphi_{t}^{\varepsilon}+u^{\varepsilon} \cdot \nabla \varphi^{\varepsilon}+\nu \Delta \varphi^{\varepsilon}=H \\
\varphi^{\varepsilon}(\cdot, T)=0,
\end{array}\right.
$$

for given functions $G, H \in H^{\infty}\left(\mathbb{R}^{3} \times[0, T]\right)$. By simple estimates, $\psi^{\varepsilon}$ and $\varphi^{\varepsilon}$ satisfy the following bounds in terms of $G$ and $H$ :

$$
\begin{aligned}
& \sup _{0 \leq \tau \leq T} \int_{\mathbb{R}^{3}}\left[\left|\psi^{\varepsilon}(x, t)\right|^{2}+\left|\nabla \psi^{\varepsilon}(x, t)\right|^{2}\right] d x+\int_{0}^{T} \int_{\mathbb{R}^{3}}\left[\left|\psi_{t}^{\varepsilon}+\nabla \psi^{\varepsilon} u^{\varepsilon}\right|^{2}+\left|D_{x}^{2} \psi^{\varepsilon}\right|^{2}\right] d x d \tau \\
& \leq C \int_{0}^{T} \int_{\mathbb{R}^{3}}|G|^{2} d x d \tau
\end{aligned}
$$

and

$$
\begin{aligned}
& \sup _{0 \leq \tau \leq T} \int_{\mathbb{R}^{3}}\left[\left|\varphi^{\varepsilon}(x, t)\right|^{2}+\left|\nabla \varphi^{\varepsilon}(x, t)\right|^{2}\right] d x+\int_{0}^{T} \int_{\mathbb{R}^{3}}\left[\left|\varphi_{t}^{\varepsilon}+\nabla \varphi^{\varepsilon} u^{\varepsilon}\right|^{2}+\left|D_{x}^{2} \varphi^{\varepsilon}\right|^{2}\right] d x d \tau \\
& \leq C \int_{0}^{T} \int_{\mathbb{R}^{3}}|H|^{2} d x d \tau
\end{aligned}
$$

We now take $\psi=\psi^{\varepsilon}$ in (4.9) and $\varphi=\varphi^{\varepsilon}$ in (4.10) respectively to obtain

$$
\begin{array}{r}
\int_{\mathbb{R}^{3}}\left(\bar{\rho} \bar{u}_{0}-\rho_{0} u_{0}\right) \cdot \psi^{\varepsilon}(x, 0) d x=\int_{0}^{T} \int_{\mathbb{R}^{3}} z \cdot G d x d \tau+\sum_{i=1}^{7} \mathcal{R}_{i}, \\
-\int_{\mathbb{R}^{3}}\left(\bar{B}_{0}-B_{0}\right) \cdot \varphi^{\varepsilon}(x, 0) d x=\int_{0}^{T} \int_{\mathbb{R}^{3}}(B-\bar{B}) \cdot H d x d \tau+\mathcal{R}_{8},
\end{array}
$$

where $z=u-\bar{u} \circ S$ and $\mathcal{R}_{1}, \ldots, \mathcal{R}_{8}$ are given by:

$$
\begin{gathered}
\mathcal{R}_{1}=\int_{0}^{T} \int_{\mathbb{R}^{3}}\left[\nabla \bar{F} \cdot\left(\psi^{\varepsilon}-\psi^{\varepsilon} \circ S^{-1}\right)+\mu \bar{\omega}_{x_{k}}^{j, k}\left(\psi^{\varepsilon}-\psi^{\varepsilon} \circ S^{-1}\right)\right] d x d \tau, \\
\mathcal{R}_{2}=\int_{0}^{T} \int_{\mathbb{R}^{3}} z \cdot\left[\left(\rho-\rho^{\varepsilon}\right) \psi_{t}^{\varepsilon}+\nabla \psi^{\varepsilon}\left(\rho u-\rho^{\varepsilon} u^{\varepsilon}\right)\right] d x d \tau, \\
\mathcal{R}_{3}=\int_{0}^{T} \int_{\mathbb{R}^{3}}(\bar{u} \circ S-\bar{u}) \cdot\left(\mu \Delta \psi^{\varepsilon}+\lambda \operatorname{div}\left(\psi^{\varepsilon}\right)\right) d x d \tau, \\
\mathcal{R}_{4}=\int_{0}^{T} \int_{\mathbb{R}^{3}}\left(1-A_{0}\right) \rho(\bar{u} \circ S) \cdot\left(\psi_{t}^{\varepsilon}+\nabla \psi^{\varepsilon} u\right) d x d \tau, \\
\mathcal{R}_{5}=\int_{0}^{T} \int_{\mathbb{R}^{3}}(P(\rho)-P(\bar{\rho})) \operatorname{div}\left(\psi^{\varepsilon}\right) d x d \tau, \\
\mathcal{R}_{6}=\int_{0}^{T} \int_{\mathbb{R}^{3}} \bar{B}_{x_{k}}^{j} \bar{B}^{k}\left(\left(\psi^{\varepsilon}\right)^{j}-\left(\psi^{\varepsilon}\right)^{j} \circ S^{-1}\right) d x d \tau \\
-\int_{0}^{T} \int_{\mathbb{R}^{3}} \frac{1}{2} \nabla\left(|\bar{B}|^{2}\right) \cdot\left(\psi^{\varepsilon}-\psi^{\varepsilon} \circ S^{-1}\right) d x d \tau, \\
\mathcal{R}_{7}=\int_{0}^{T} \int_{\mathbb{R}^{3}} \frac{1}{2}\left(|B|^{2}-|\bar{B}|^{2}\right) \operatorname{div}\left(\psi^{\varepsilon}\right) d x d \tau \\
-\int_{0}^{T} \int_{\mathbb{R}^{3}}\left(B^{j} B-\bar{B}^{j} \bar{B}\right) \cdot \psi^{\varepsilon} d x d \tau,
\end{gathered}
$$


and

$$
\begin{aligned}
\mathcal{R}_{8}= & \int_{0}^{T} \int_{\mathbb{R}^{3}} \nabla\left(\varphi^{\varepsilon}\right)^{j}(\bar{B}-B) \bar{B}^{j} d x d \tau+\int_{0}^{T} \int_{\mathbb{R}^{3}} \nabla\left(\varphi^{\varepsilon}\right)^{j}\left(\bar{u}^{j}-u^{j}\right) B d x d \tau \\
& +\int_{0}^{T} \int_{\mathbb{R}^{3}} \nabla\left(\varphi^{\varepsilon}\right)^{j}(u-\bar{u}) \bar{B}^{j} d x d \tau .
\end{aligned}
$$

Our main goal is to estimate the terms $\mathcal{R}_{1}, \ldots, \mathcal{R}_{8}$ and the terms on the left sides of (4.13)-(4.14) and then take the limit as $\varepsilon \rightarrow 0$. Most of the analysis are reminiscent of those given in Hoff [12] except the terms $\mathcal{R}_{1}, \mathcal{R}_{3}, \mathcal{R}_{6}, \mathcal{R}_{7}$ and $\mathcal{R}_{8}$.

Following the steps given in [12, using Proposition 4.2 and applying the bound (4.11) on $\psi^{\varepsilon}$, we are ready to obtain:

$$
\begin{aligned}
& \left|\int_{\mathbb{R}^{3}}\left(\bar{\rho} \bar{u}_{0}-\rho_{0} u_{0}\right) \cdot \psi^{\varepsilon}(x, 0) d x\right|+\left|\int_{\mathbb{R}^{3}}\left(\bar{B}_{0}-B_{0}\right) \cdot \varphi^{\varepsilon}(x, 0) d x\right| \\
& \leq\left\|\rho_{0} u_{0}-\bar{\rho}_{0} \bar{u}_{0}\right\|_{L^{2}}\left(\int_{0}^{T} \int_{\mathbb{R}^{3}}|G|^{2} d x d \tau\right)^{\frac{1}{2}}+\left\|B_{0}-\bar{B}_{0}\right\|_{L^{2}}\left(\int_{0}^{T} \int_{\mathbb{R}^{3}}|H|^{2} d x d \tau\right)^{\frac{1}{2}} \\
& \left|\mathcal{R}_{4}\right| \leq C T^{\frac{1}{2}}\left[\left\|\rho_{0}-\bar{\rho}_{0}\right\|_{L^{2}}+\left(\int_{0}^{T} \int_{\mathbb{R}^{3}}|z|^{2} d x d \tau\right)^{\frac{1}{2}}\right]\left(\int_{0}^{T} \int_{\mathbb{R}^{3}}|G|^{2} d x d \tau\right)^{\frac{1}{2}} \\
& \lim _{\varepsilon \rightarrow 0} \mathcal{R}_{2}=0
\end{aligned}
$$

and under the assumption (1.29) on pressure $P$, the following estimate holds

$$
\begin{aligned}
\left|\mathcal{R}_{5}\right| & \leq \int_{0}^{T} \int_{\mathbb{R}^{3}} K\left|(\rho-\bar{\rho}) \operatorname{div}\left(\psi^{\varepsilon}\right)\right| d x d \tau \\
& \leq C T^{\frac{1}{2}}\left[\left\|\rho_{0}-\bar{\rho}_{0}\right\|_{L^{2}}+\left(\int_{0}^{T} \int_{\mathbb{R}^{3}}|z|^{2} d x d \tau\right)^{\frac{1}{2}}\right]\left(\int_{0}^{T} \int_{\mathbb{R}^{3}}|G|^{2} d x d \tau\right)^{\frac{1}{2}} .
\end{aligned}
$$

We now give the estimates $\mathcal{R}_{1}, \mathcal{R}_{3}, \mathcal{R}_{6}, \mathcal{R}_{7}$ and $\mathcal{R}_{8}$ as follows. To estimate $\mathcal{R}_{1}$, modulo the vorticity $\omega$, we obtain that

$$
\begin{aligned}
\left|\mathcal{R}_{1}\right| \leq & C\left(\int_{0}^{T} \int_{\mathbb{R}^{3}}|z|^{2} d x d \tau\right)^{\frac{1}{2}} \int_{0}^{T} \tau^{\frac{1}{2}}\|\nabla \bar{F}(\cdot, \tau)\|_{L^{4}}\left\|\nabla \psi^{\varepsilon}(\cdot, t)\right\|_{L^{4}} d \tau \\
\leq & C\left(\int_{0}^{T} \int_{\mathbb{R}^{3}}|z|^{2} d x d \tau\right)^{\frac{1}{2}}\left(\int_{0}^{T} \int_{\mathbb{R}^{3}}|G|^{2} d x d \tau\right)^{\frac{1}{2}}\left(\int_{0}^{T} \tau^{\frac{4}{5}}\|\nabla \bar{F}(\cdot, \tau)\|_{L^{4}}^{\frac{8}{5}} d \tau\right)^{\frac{5}{8}} \\
& \times\left(\int_{0}^{T} \int_{\mathbb{R}^{3}}\left|D_{x}^{2} \psi^{\varepsilon}\right|^{2} d x d \tau\right)^{\frac{3}{8}} .
\end{aligned}
$$

To bound the term involving $\bar{F}$ as above, we use (4.1)-(4.2) to obtain

$$
\int_{0}^{T} \tau^{\frac{4}{5}}\|\nabla \bar{F}(\cdot, \tau)\|_{L^{4}}^{\frac{8}{5}} d \tau \leq \int_{0}^{T} \tau^{\frac{4}{5}}\left(\int_{\mathbb{R}^{3}}|\dot{\bar{u}}|^{4} d x+\int_{\mathbb{R}^{3}}|\nabla \bar{B}|^{4}|\bar{B}|^{4} d x\right)^{\frac{3}{5}} d \tau .
$$

The first integral on the right side of (4.19) is bounded by

$$
\begin{aligned}
& \int_{0}^{T} \tau^{\frac{4}{5}}\left(\int_{\mathbb{R}^{3}}|\dot{\bar{u}}|^{4} d x\right)^{\frac{3}{5}} d \tau \\
& \leq C \int_{0}^{T} \tau^{\frac{4}{5}}\left(\int_{\mathbb{R}^{3}}|\dot{\bar{u}}|^{2} d x\right)^{\frac{1}{5}}\left(\int_{\mathbb{R}^{3}}|\nabla \dot{\bar{u}}|^{2} d x\right)^{\frac{3}{5}} d \tau \\
& \leq C\left(\int_{0}^{T} \tau^{4 s-3} d \tau\right)^{\frac{1}{5}}\left(\int_{0}^{T} \tau^{1-s} \int_{\mathbb{R}^{3}}|\dot{\bar{u}}|^{2} d x d \tau\right)^{\frac{1}{5}}\left(\int_{0}^{T} \tau^{1-s} \int_{\mathbb{R}^{3}}|\dot{\bar{u}}|^{2} d x d \tau\right)^{\frac{1}{5}} \leq C T^{\frac{4 s-2}{5}},
\end{aligned}
$$


where the last inequality holds by the bounds (1.26) and (4.1)-4.2), and the assumption that $s>\frac{1}{2}$. On the other hand, to bound the term involving $B \cdot \nabla B$ in (4.19), we have

$$
\begin{aligned}
& \int_{0}^{T} \tau^{\frac{4}{5}}\left(\int_{\mathbb{R}^{3}}|\nabla B|^{4}|B|^{4} d x\right)^{\frac{2}{5}} d \tau \\
& \leq C\left(\int_{0}^{T} \tau^{4 s-3} d \tau\right)^{\frac{1}{5}}\left(\int_{0}^{T} \tau^{1-s} \int_{\mathbb{R}^{3}}|\nabla B|^{2}|B|^{2} d x d \tau\right)^{\frac{1}{5}} \\
& \quad \times\left(\int_{0}^{T} \tau^{2-s} \int_{\mathbb{R}^{3}}|\nabla B|^{4} d x d \tau+\int_{0}^{T} \tau^{2-s} \int_{\mathbb{R}^{3}}|\Delta B|^{2}|B|^{2} d x d \tau\right)^{\frac{3}{5}} .
\end{aligned}
$$

Following the proof of the bound (2.25) in Lemma 2.9, we use (4.1)-4.2) to obtain

$$
\int_{0}^{T} \tau^{1-s} \int_{\mathbb{R}^{3}}|\nabla B|^{2}|B|^{2} d x d \tau+\int_{0}^{T} \tau^{2-s} \int_{\mathbb{R}^{3}}|\nabla B|^{4} d x d \tau \leq C .
$$

Using the magnetic field equation (1.3), we also have

$$
\begin{aligned}
& \int_{0}^{T} \tau^{2-s} \int_{\mathbb{R}^{3}}|\Delta B|^{2}|B|^{2} d x d \tau \\
& \leq C\left(\int_{0}^{T} \int_{\mathbb{R}^{3}} \tau^{2-s}\left|B_{t}\right|^{2}|B|^{2} d x d \tau+\int_{0}^{T} \int_{\mathbb{R}^{3}} \tau^{2-s}\left(|\nabla u|^{2}|B|^{2} d x d \tau+|\nabla B|^{2}|u|^{2}\right)|B|^{2} d x d \tau\right) \\
& \leq C+C \int_{0}^{T} \int_{\mathbb{R}^{3}} \tau^{2-s}\left|B_{t}\right|^{2}|B|^{2} d x d \tau,
\end{aligned}
$$

and also

$$
\begin{aligned}
& \int_{0}^{T} \int_{\mathbb{R}^{3}} \tau^{2-s}\left|B_{t}\right|^{2}|B|^{2} d x d \tau \\
& \leq \int_{0}^{T} \tau^{2-s}\left(\int_{\mathbb{R}^{3}}\left|B_{t}\right|^{3} d x\right)^{\frac{2}{3}}\left(\int_{\mathbb{R}^{3}}|B|^{6} d x\right)^{\frac{1}{3}} d \tau \\
& \leq C\left(\sup _{0 \leq \tau \leq T} \int_{\mathbb{R}^{3}}|B|^{6} d x\right)^{\frac{1}{3}}\left(\int_{0}^{T} \int_{\mathbb{R}^{3}} \tau^{2-s}\left|\nabla B_{t}\right|^{2} d x d \tau\right)^{\frac{1}{2}}\left(\int_{0}^{T} \int_{\mathbb{R}^{3}} \tau^{1-s}\left|B_{t}\right|^{2} d x d \tau\right)^{\frac{1}{2}} \\
& \leq C .
\end{aligned}
$$

Combining the above estimates, we conclude from (4.19) that

$$
\int_{0}^{T} \tau^{\frac{4}{5}}\|\nabla \bar{F}(\cdot, \tau)\|_{L^{4}}^{\frac{8}{5}} d \tau \leq C T^{\frac{2 s-1}{4}}
$$

and we obtain the estimate on $\mathcal{R}_{1}$

$$
\left|\mathcal{R}_{1}\right| \leq C T^{\frac{2 s-1}{4}}\left(\int_{0}^{T} \int_{\mathbb{R}^{3}}|z|^{2} d x d \tau\right)^{\frac{1}{2}}\left(\int_{0}^{T} \int_{\mathbb{R}^{3}}|G|^{2} d x d \tau\right)^{\frac{1}{2}} .
$$

In particular, for $\left[t_{1}, t_{2}\right] \subseteq[0, T]$, if we define

$$
\mathcal{R}_{1}\left(t_{1}, t_{2}\right)=\int_{t_{1}}^{t_{2}} \int_{\mathbb{R}^{3}}\left[\nabla \bar{F} \cdot\left(\psi^{\varepsilon}-\psi^{\varepsilon} \circ S^{-1}\right)+\mu \bar{\omega}_{x_{k}}^{j, k}\left(\psi^{\varepsilon}-\psi^{\varepsilon} \circ S^{-1}\right)\right] d x d \tau,
$$

then we also have

$$
\left|\mathcal{R}_{1}\left(t_{1}, t_{2}\right)\right| \leq C\left|t_{2}-t_{1}\right|^{\frac{2 s-1}{4}}\left(\int_{0}^{t_{2}} \int_{\mathbb{R}^{3}}|z|^{2} d x d \tau\right)^{\frac{1}{2}}\left(\int_{0}^{t_{2}} \int_{\mathbb{R}^{3}}|G|^{2} d x d \tau\right)^{\frac{1}{2}}
$$


with $C$ being independent of $t_{1}, t_{2}$ and $G$. The term $\mathcal{R}_{3}$ can be bounded in a similar way as $\mathcal{R}_{1}$.

To estimate $\mathcal{R}_{6}$, in view of the definition of $\mathcal{R}_{6}$, we first consider the term $\int_{0}^{T} \int_{\mathbb{R}^{3}} \bar{B}_{x_{k}}^{j} \bar{B}^{k}\left(\left(\psi^{\varepsilon}\right)^{j}-\left(\psi^{\varepsilon}\right)^{j} \circ S^{-1}\right)$ which can be bounded as follows.

$$
\begin{aligned}
& \left|\int_{0}^{T} \int_{\mathbb{R}^{3}} \bar{B}_{x_{k}}^{j} \bar{B}^{k}\left(\left(\psi^{\varepsilon}\right)^{j}-\left(\psi^{\varepsilon}\right)^{j} \circ S^{-1}\right) d x d \tau\right| \\
& \leq C \int_{0}^{T} \int_{\mathbb{R}^{3}}\left|\nabla \bar{B}\|\bar{B}\| \psi^{\varepsilon}(x, \tau)-\psi^{\varepsilon}\left(S^{-1}(x, \tau), \tau\right)\right| d x d \tau \\
& \leq C\left(\int_{0}^{T} \int_{\mathbb{R}^{3}}|z|^{2} d x d \tau\right)^{\frac{1}{2}} \int_{0}^{T} t^{\frac{1}{2}}\|\nabla \bar{B}(\cdot, \tau)\|_{L^{4}}\left\|\nabla \psi^{\varepsilon}(\cdot, \tau)\right\|_{L^{4}} d t \\
& \leq C\left(\int_{0}^{T} \int_{\mathbb{R}^{3}}|z|^{2} d x d \tau\right)^{\frac{1}{2}}\left(\int_{0}^{T} \int_{\mathbb{R}^{3}}|G|^{2} d x d \tau\right)^{\frac{1}{2}}\left(\int_{0}^{T} \tau^{\frac{4}{5}}\|\nabla \bar{B}(\cdot, \tau)\|_{L^{4}}^{\frac{8}{5}} d \tau\right)^{\frac{5}{8}} .
\end{aligned}
$$

The term $\int_{0}^{T} \tau^{\frac{4}{5}}\|\nabla \bar{B}(\cdot, t)\|_{L^{4}}^{\frac{8}{5}} d \tau$ can be bounded in a similar way as $\int_{0}^{T} \tau^{\frac{4}{5}}\|\nabla \bar{F}(\cdot, t)\|_{L^{4}}^{\frac{8}{5}} d \tau$, hence we obtain

$$
\begin{aligned}
& \left|\int_{0}^{T} \int_{\mathbb{R}^{3}} \bar{B}_{x_{k}}^{j} \bar{B}^{k}\left(\left(\psi^{\varepsilon}\right)^{j}-\left(\psi^{\varepsilon}\right)^{j} \circ S^{-1}\right) d x d \tau\right| \\
& \leq C T^{\frac{2 s-1}{4}}\left(\int_{0}^{T} \int_{\mathbb{R}^{3}}|z|^{2} d x d \tau\right)^{\frac{1}{2}}\left(\int_{0}^{T} \int_{\mathbb{R}^{3}}|G|^{2} d x d \tau\right)^{\frac{1}{2}} .
\end{aligned}
$$

On the other hand, the term $\int_{0}^{T} \int_{\mathbb{R}^{3}} \frac{1}{2} \nabla\left(|\bar{B}|^{2}\right) \cdot\left(\psi-\psi \circ S^{-1}\right) d x d \tau$ in the definition of $\mathcal{R}_{6}$ can be treated similarly, and hence we conclude

$$
\left|\mathcal{R}_{6}\right| \leq C T^{\frac{2 s-1}{4}}\left(\int_{0}^{T} \int_{\mathbb{R}^{3}}|z|^{2} d x d \tau\right)^{\frac{1}{2}}\left(\int_{0}^{T} \int_{\mathbb{R}^{3}}|G|^{2} d x d \tau\right)^{\frac{1}{2}}
$$

and in particular

$$
\left|\mathcal{R}_{6}\left(t_{1}, t_{2}\right)\right| \leq C\left|t_{2}-t_{1}\right|^{\frac{2 s-1}{4}}\left(\int_{0}^{t_{2}} \int_{\mathbb{R}^{3}}|z|^{2} d x d \tau\right)^{\frac{1}{2}}\left(\int_{0}^{t_{2}} \int_{\mathbb{R}^{3}}|G|^{2} d x d \tau\right)^{\frac{1}{2}},
$$

where $\left[t_{1}, t_{2}\right] \subseteq[0, T]$ and $\mathcal{R}_{6}\left(t_{1}, t_{2}\right)$ is given by

$$
\mathcal{R}_{6}\left(t_{1}, t_{2}\right)=\int_{t_{1}}^{t_{2}} \int_{\mathbb{R}^{3}}\left[\bar{B}_{x_{k}}^{j} \bar{B}^{k}\left(\left(\psi^{\varepsilon}\right)^{j}-\left(\psi^{\varepsilon}\right)^{j} \circ S^{-1}\right)-\frac{1}{2} \nabla\left(|\bar{B}|^{2}\right) \cdot\left(\psi^{\varepsilon}-\psi^{\varepsilon} \circ S^{-1}\right)\right] d x d \tau .
$$

To estimate $\mathcal{R}_{7}$, we can readily obtain the bound as follows:

$$
\left|\mathcal{R}_{7}\right| \leq C\left(\int_{0}^{T} \int_{\mathbb{R}^{3}}|B-\bar{B}|^{2} d x d \tau\right)^{\frac{1}{2}}\left(\int_{0}^{T} \int_{\mathbb{R}^{3}}\left(|B|^{6}+|\bar{B}|^{6}\right) d x d \tau\right)^{\frac{1}{6}}\left(\int_{0}^{T} \int_{\mathbb{R}^{3}}\left|\nabla \psi^{\varepsilon}\right|^{3} d x d \tau\right)^{\frac{1}{3}}
$$

which gives

$$
\left|\mathcal{R}_{7}\right| \leq C T^{\frac{1}{3}}\left(\int_{0}^{T} \int_{\mathbb{R}^{3}}|G|^{2} d x d \tau\right)^{\frac{1}{2}}
$$


Similarly, for the term $\mathcal{R}_{8}$, using the bound (4.12) we have the estimate

$$
\begin{aligned}
\left|\mathcal{R}_{8}\right| \leq & C\left(\int_{0}^{T} \int_{\mathbb{R}^{3}}\left(|u-\bar{u}|^{2}+|B-\bar{B}|^{2}\right) d x d \tau\right)^{\frac{1}{2}} \\
& \times\left(\int_{0}^{T} \int_{\mathbb{R}^{3}}\left(|B|^{6}+|\bar{B}|^{6}\right) d x d \tau\right)^{\frac{1}{6}}\left(\int_{0}^{T} \int_{\mathbb{R}^{3}}\left|\nabla \varphi^{\varepsilon}\right|^{3} d x d \tau\right)^{\frac{1}{3}} \\
\leq & C T^{\frac{1}{3}}\left(\int_{0}^{T} \int_{\mathbb{R}^{3}}|H|^{2} d x d \tau\right)^{\frac{1}{2}} .
\end{aligned}
$$

Summarizing the estimates (4.15), (4.16), (4.17), (4.18), (4.20), (4.21), (4.22) and (4.23), we arrive at

$$
\begin{aligned}
& \left|\int_{0}^{T} \int_{\mathbb{R}^{3}} z \cdot G d x d \tau\right| \\
& \leq C\left[M_{0}\left(\int_{0}^{T} \int_{\mathbb{R}^{3}}|G|^{2} d x d \tau\right)^{\frac{1}{2}}+\left|\mathcal{R}_{1}(0, T)\right|+\left|\mathcal{R}_{6}(0, T)\right|\right],
\end{aligned}
$$

and

$$
\left|\int_{0}^{T} \int_{\mathbb{R}^{3}}(B-\bar{B}) \cdot H d x d \tau\right| \leq C M_{0}\left(\int_{0}^{T} \int_{\mathbb{R}^{3}}|H|^{2} d x d \tau\right)^{\frac{1}{2}}
$$

where $M_{0}$ is given by

$$
M_{0}=\left\|\rho_{0}-\bar{\rho}_{0}\right\|_{L^{2}}+\left\|\rho_{0} u_{0}-\bar{\rho}_{0} \bar{u}_{0}\right\|_{L^{2}}+T^{\delta}\left(\int_{0}^{T} \int_{\mathbb{R}^{3}}|z|^{2} d x d \tau\right)^{\frac{1}{2}}
$$

for some $\delta>0$, and $C>0$ is now fixed. Following the analysis given in Hoff [12, pp. 1758-1759], there exists a small time $\tilde{\tau}>0$ such that

$$
\left(\int_{0}^{\tilde{\tau}} \int_{\mathbb{R}^{3}}|z|^{2} d x d \tau\right)^{\frac{1}{2}} \leq 2 C M_{0}
$$

and consequently

$$
\left|\mathcal{R}_{1}(0, \tilde{\tau})\right|+\left|\mathcal{R}_{6}(0, \tilde{\tau})\right| \leq M_{0}\left(\int_{0}^{\tilde{\tau}} \int_{\mathbb{R}^{3}}|G|^{2} d x d \tau\right)^{\frac{1}{2}} .
$$

By applying (4.24) with $T$ replaced by $2 \tilde{\tau}$, we get

$$
\left(\int_{0}^{2 \tilde{\tau}} \int_{\mathbb{R}^{3}}|z|^{2} d x d \tau\right)^{\frac{1}{2}} \leq 4 C M_{0}
$$

Since $\tilde{\tau}>0$ is fixed, we can exhaust the interval $[0, T]$ in finitely many steps to obtain that

$$
\left(\int_{0}^{T} \int_{\mathbb{R}^{3}}|z|^{2} d x d \tau\right)^{\frac{1}{2}} \leq C M_{0}
$$

for some new constant $C>0$. Hence the term $T^{\delta}\left(\int_{0}^{T} \int_{\mathbb{R}^{3}}|z|^{2} d x d \tau\right)^{\frac{1}{2}}$ can be eliminated from the definition of $M_{0}$ by a Gronwäll-type argument. Therefore we conclude that

$$
\begin{aligned}
\left|\int_{0}^{T} \int_{\mathbb{R}^{3}} z \cdot G d x d \tau\right| & \leq C M_{0}\left(\int_{0}^{T} \int_{\mathbb{R}^{3}}|G|^{2} d x d \tau\right)^{\frac{1}{2}} \\
\left|\int_{0}^{T} \int_{\mathbb{R}^{3}}(B-\bar{B}) \cdot H d x d \tau\right| & \leq C M_{0}\left(\int_{0}^{T} \int_{\mathbb{R}^{3}}|H|^{2} d x d \tau\right)^{\frac{1}{2}} .
\end{aligned}
$$


Since (4.25) and (4.26) hold for any $G, H \in H^{\infty}\left(\mathbb{R}^{3} \times[0, T]\right)$, it shows that both $\|z\|_{L^{2}\left([0, T] \times \mathbb{R}^{3}\right)}$ and $\|B-\bar{B}\|_{L^{2}\left([0, T] \times \mathbb{R}^{3}\right)}$ are bounded by $M_{0}$. Finally, using the bound (4.3) on $\nabla \bar{u}$,

$$
\begin{aligned}
\int_{0}^{T} \int_{\mathbb{R}^{3}}|\bar{u}-\bar{u} \circ S|^{2} d x d \tau & \leq \int_{0}^{T}\|\nabla \bar{u}(\cdot, \tau)\|_{L^{\infty}}^{2} \int_{\mathbb{R}^{3}}|x-S(x, \tau)|^{2} d x d \tau \\
& \leq C \int_{0}^{T} \int_{\mathbb{R}^{3}}|z|^{2} d x d \tau,
\end{aligned}
$$

and hence (1.30) follows. We finish the proof of Theorem 1.5

\section{REFERENCES}

[1] Alfvén, H. 1942, Existence of electromagnetic-hydrodynamic waves, Nature, vol. 150, pp. 405.

[2] Bahouri, H., Chemin, J.-Y., Equations de transport relatives des champs de vecteurs nonLipschitziens et mecanique des fluides, Arch. Rational Mech. Anal. 127(2), 159-181 (1994).

[3] Biskamp, D. Nonlinear Magnetohydrodynamics, Cambridge University Press, 1997.

[4] Cabannes, H., Theoretical Magneto-Fluid Dynamics, Academic Press, New York, London, 1970.

[5] Davidson, P., An Introduction to Magnetohydrodynamics, Cambridge University Press, 2001.

[6] Ducomet, B., Feireisl, E. 2006, The equations of Magnetohydrodynamics: On the interaction between matter and radiation in the evolution of gaseous stars, Commun. Math. Phys, 226, pp. 595-629.

[7] Feireisl, E., Compressible Navier-Stokes equations with a non-monotone pressure law, J. Diff. Eqns, 184 (2002), pp. 97-108.

[8] Feireisl, E., Dynamics of Viscous Compressible Fluids, Oxford Lecture Series in Mathematics and its Applications, 26. Oxford University Press, Oxford, 2004.

[9] Freistühler, H. 1993, Contributions to the mathematical theory of magnetohydrodynamic shock waves, Nonlinear evolutionary partial differential equations, pp.175-187.

[10] Hoff, D., Global solutions of the Navier-Stokes equations for multidimensional, compressible flow with discontinuous initial data, J. Diff. Eqns. 120, no. 1 (1995), 215-254.

[11] Hoff, D., Dynamics of Singularity Surfaces for Compressible, Viscous Flows in Two Space Dimensions, Communications on Pure and Applied Mathematics, Vol. LV (2002), 1365-1407.

[12] Hoff, D., Uniqueness of weak solutions of the Navier-Stokes equations of multidimensional compressible flow, SIAM J. Math. Anal 37, no. 6 (2006), 1742-1760.

[13] Hoff, D. 2011, Existence of Solutions to a Model for Sparse, One-dimensional Fluids, J. Diff. Eqns. 250, no. 2, pp. 1083-1113.

[14] Hoff, D. and Serre, D. 1991, The failure of continuous dependence on initial data for the Navier-Stokes equations of compressible flow, SIAM J. Appl. Math. 51, pp. 887-898.

[15] Hoff, D. and Santos, M. 2008, Lagrangean Structure and Propagation of Singularities in Multidimensional Compressible Flow, Arch. Rational Mech. Anal. 188, pp. 509-543.

[16] P.L. Lions, Mathematical Topics in Fluid Mechanics, vol. 2, Oxford Lecture Series in Mathematics, 10 (1998).

[17] $\mathrm{Hu}, \mathrm{X}$. and Wang, D., Global solutions to the three-dimensional full compressible magnetohydrodynamic flows, Comm. Math. Phys. 283, no. 1 (2008), 255-284.

[18] Hu, X. and Wang, D., Global existence and large-time behavior of solutions to the threedimensional equations of compressible magnetohydrodynamic flows, Arch. Ration. Mech. Anal. 197, no. 1 (2010), 203-238.

[19] Kawashima, S., Systems of a hyperbolic-parabolic composite type, with applications to the equations of magnetohydrodynamics, Ph. D. Thesis, Kyoto University, (1983).

[20] Sart, R., Existence of finite energy weak solutions for the equations MHD of compressible fluids, Appl. Anal. 88, no. 3 (2009), 357-379.

[21] Stein, E. M., Singular Integrals and Differentiability Properties of Functions, Princeton Univ. Press, 1970.

[22] Suen, A. and Hoff, D., Global low-energy weak solutions of the equations of $3 D$ compressible magnetohydrodynamics, Arch. Rational Mechanics Ana. 205 (2012), no. 1, 27-58.

[23] Ziemer, W., Weakly differentiable functions, Springer-Verlag, 1989. 
Department of Mathematics and Information Technology, The Education University OF Hong Kong, Hong Kong

Email address: acksuen@eduhk.hk 